\title{
ON THE POSSIBLE EXISTENCE OF NON-FISHER UNIVERSALITY CLASSES
}

\author{
A.I. IVANYTSKYI, K.A. BUGAEV
}

Bogolyubov Institute for Theoretical Physics, Nat. Acad. of Sci. of Ukraine

(14b, Metrolohichna Str., Kyiv 03680, Ukraine; e-mail:

PACS 25.75.-q, 25.75.Nq

a-iv_@ukr. net, bugaev@ th. physik. uni-frankfurt. de)

The critical exponents $\alpha, \alpha^{\prime}, \beta, \gamma^{\prime}$, and $\delta$ of the model of quarkgluon bags with surface tension are found as functions of the most general model parameters. Two versions of the model that generate the phase diagram of the strongly interacting matter with critical or tricritical endpoint, respectively, are considered. The analysis of the relations between the critical exponents (scaling laws) shows that the scaling can be violated in a general case. The question whether it is possible to restore the scaling laws with the help of the Fisher definition of the $\alpha_{s}^{\prime}$ exponent and its generalizations $\alpha_{c}^{\prime}$ and $\alpha_{m}^{\prime}$ is studied. It is shown that the Fisher scaling relation can be recovered with the help of the generalizations $\alpha_{c}^{\prime}$ and $\alpha_{m}^{\prime}$, whereas no definition of the $\alpha^{\prime}$ index is able to recover the Griffiths scaling relation in its traditional form. It is explicitly demonstrated that the additional condition $\alpha=\alpha^{\prime}$ is not sufficient to restore the Griffiths scaling relation in the traditional form. A generalization of this scaling relation which is valid for all known models is suggested. The obtained results allow us to conclude on the possible existence of the non-Fisher universality classes, for which the traditional scaling relations can be violated, whereas the generalized scaling laws can be established.

\section{Introduction}

While studying the thermodynamical properties of certain physical systems, the researchers are faced with extremely interesting phenomena such as various critical ones. The starting point of studies in this field was the discovery of "the absolute boiling temperature" made by D.I. Mendeleev, at which the surface tension of two coexisting phases disappears at a phase transition (PT) of the liquid-vapor type. Hence, these phases become nondistinguishable. In this case, the physical system exists in the critical state, and the corresponding point of the phase diagram is called the critical point (CP). By approaching this point along the PT curve, the difference of the densities of two phases decreases up to its vanishing. The studies of thermodynamical properties of magnetic systems have also demonstrated the existence of $\mathrm{CP}$, at which the spontaneous magnetization of the system disappears.
The further development of ideas of critical phenomena was related to the experimental determination of the thermodynamical quantities that characterize CPs of some physical system: critical pressure, temperature, and density. As a qualitative breakthrough, we mention the comprehension of the fact that different physical systems manifest the extraordinary similarity of their behavior in the course of approaching CP. For example, the existence of some universal curve for a PT of the liquid-vapor type in the coordinates $\rho-T$ normalized to the critical values was demonstrated in work [1]. The measurements on eight substances ( $\mathrm{Ne}, \mathrm{Ar}, \mathrm{Kr}, \mathrm{Xe}, \mathrm{N}_{2}$, $\mathrm{O}_{2}, \mathrm{CO}$, and $\mathrm{CH}_{4}$ ) testified to the existence of a similarity in the critical behavior of various physical systems, which belong to a single universality class. It is natural to ask how the universality class for a certain substance can be determined. The possibility of the existence of different universality classes is indicated by the fact that many magnetic systems quite different by their nature from simple fluids demonstrate also the universality of the critical behavior.

The first attempt to determine the universality class of certain physical systems was based on the analysis of the properties of their CPs. Since the CPs are described by critical exponents, the study of their values and the scaling relations between them $[2,3]$ became the foundation of the proposed classification. The Fisher theorem proved in these works asserts that the scaling relations for physical systems with most general thermodynamical properties have some specific form, and the systems themselves fall in a single universality class. However, the situation concerning the scaling relations between critical exponents of real substances and some physical models satisfying the requirements of the Fisher theorem turned out to be not so simple. For example, the scaling relations hold not in all the cases even in even in the limits of the experimental accuracy for some simple liquids [4-8] and three-dimensional Ising model [9]. Moreover, it was shown in [3] long time ago that the standard definition of the critical exponent $\alpha^{\prime}$ along the critical isochore can lead to the violation of the scal- 
ing laws even for the models satisfying the requirements of the Fisher theorem. Thus, the extremely important question arose: Does the reason for the violation of the scaling laws in the Fisher form for some exactly solvable models lie in the incorrectness of the definitions of some critical exponents (e.g., $\alpha^{\prime}$ ) or in the insufficient degree of generality of the Fisher theorem? In turn, the latter can testify to the existence of non-Fisher universality classes, for which this theorem is not valid. In the present work, we will analyze different definitions of critical exponents in order to answer the question about the validity of the scaling relations in the traditional form.

The indicated problems of the scaling systematization of universality classes caused the development of an alternative approach [10] based on the analysis of the quite general requirements of stability of the critical state. Their analysis allowed one to separate four basic types of CPs, which correspond to certain general universality classes of the critical behavior of a system. As one of the advantages of this approach, we mention the clear classification of a number of statistical models and real physical systems. However, it is worth noting that the absence of the predictions as for the form of scaling laws for a certain system is a significant drawback of this approach. This circumstance does not allow one to reject the Fisher classification, because namely the identity of critical exponents related to the behavior of systems in a neighborhood of CP is the experimentally observed manifestation of the fact that the systems belong to a single universality class. The attempt to partially solve this problem was made in work [11], where the role of the condition for the scaling relations in the Fisher form to be valid was played by the equality of the critical exponents $\alpha$ and $\alpha^{\prime}$. The validity of this condition together with requirements of the Fisher theorem would be sufficient in order to refer some physical system to Fisher's universality classes. Just therefore, it is very important to verify the validity of the scaling laws in the case where $\alpha=\alpha^{\prime}$. In what follows, we will demonstrate the existence of examples, where this equality together with requirements of the Fisher theorem do not yield all three most general scaling relations. However, it will be shown that the physically substantiated definition of the exponent $\alpha_{c}^{\prime}$ allows one to restore the Fisher relation, rather than the Griffiths relation. Moreover, since the latter can be violated in the traditional form for all available definitions of the exponent $\alpha^{\prime}$, this relation must be reformulated. Just these circumstances testify to, in our opinion, the possibility of the existence of non-Fisher universality classes.
The indicated difficulties of the definition of the universality class for a certain physical system must be removed due to the reasons of a purely practical character, which are related to the extremely wide use of the hypothesis of similarity, rather than due to the abstract interest. The bright example of the application of this hypothesis is given by quantum chromodynamics (QCD). It is assumed, though is not proved, that QCD in the case of $2+1$ quark flavors belongs to the universality class of three-dimensional $\mathrm{O}(4)$ spin systems [12-14]. However, according to the contemporary state of art, the quarkgluon plasma (QGP) in the deconfinement PT region is a strongly interacting liquid [15]. This circumstance complicates strongly the determination of the universality class of QCD, on the one hand, and makes it extremely important, on the other hand. The presented example demonstrates that it is very important to clarify whether the universality classes different from the Fisher one can exist. Indeed, the question about the admissibility to use the hypothesis of similarity stops to be trivial in this case, as was considered earlier. On the basis of the schemes developed in [2,3] and [10,11], we will demonstrate the possibility of the existence of universality classes different from the Fisher one.

We will study the posed questions on the basis of the analysis of the critical exponents at CPs of the strongly interacting matter and the scaling relations between them. Unfortunately, QCD and even its lattice representation do not allow us to make it. Just therefore, the further analysis will be made with the help of one of the phenomenological models $[15,16]$, which have recently acquired the extraordinary meaning. The most popular models of this type are the Nambu-Jona-Lasinio model [17], its modification on the basis of Polyakov's loops [18, 19], and the quark-meson model [20], which are mean-field models. Another direction of the development of the phenomenological approach is related to the exactly solvable model of a gas of bags (MGB) [21] (one of the modern versions of this model is given in work [22]). MGB involves the physically inadequate mechanism of generation of the tricritical point and, therefore, cannot be considered as a reliable source of the information about the thermodynamical properties of QCD. Another version of MGB is the model of quark-gluon bags (QGBM) with surface tension [23]. The mechanism of generation of $\mathrm{CP}$ in it is the same as that in the model of statistical multifragmentation (MSMF) [24] and the Fisher droplet model (FDM) [25]. It is typical of PT of the liquid-vapor type and consists in the vanishing of the coefficient of surface tension (CST) at CP, which leads to the nondistinguishability of two phases. The 
extremely important advantage of QGBM with surface tension consists in the fact that it is an exactly solvable model, rather than a mean-field one. Just therefore, QGBM allows one to study the thermodynamical properties of a strongly interacting matter, by basing on the first principles of statistical mechanics. In addition, it was first shown in the mentioned work that QGBM with surface tension has a high generality and can describe the critical exponents of a number of known universality classes including the simple fluids, Ising model, and $\mathrm{O}(2)-, \mathrm{O}(3)-$, and $\mathrm{O}(4)$-symmetric spin systems. From the viewpoint of the present work, the basically significant circumstance is the fact that QGBM with surface tension corresponds fully to requirements of the Fisher theorem and, hence, can be useful in searching the answer to the question about the possibility of the existence of non-Fisher universality classes.

The present work is organized in the following way. Section 2 gives the brief description of QGBM with surface tension and its versions. Details of calculations of the critical exponents of this model are presented in Section 3. Section 4 is devoted to the analysis of the scaling relations between critical exponents of QGBM and to the possibility to satisfy them with the help of an alternative definition of the exponent $\alpha^{\prime}$. The possibility of the existence of non-Fisher universality classes is also discussed in this section. The conclusions are presented in Section 5.

\section{QGBM with Surface Tension}

QGBM with surface tension has two versions: the model with tricritical point (TCP) [26] and the model with CP [27]. In what follows, we denote them as M1 and M2, respectively. Since they are significantly similar to each other, it is expedient firstly to present their common description and only then to consider each model separately. For the first time, the exact analytic solution of QGBM with surface tension was obtained in [23]. The physical degrees of freedom of this model are hadrons and bags in QGP. Their interaction is taken into account in the same way as that in MGB. The great number of the sorts of constituents allows us to consider the attraction between them so, as it was made in the original model of statistical bootstrap [28]. At the same time, a repulsion of the van der Waals type is a consequence of the interaction of the hard cores of hadrons and bags in QGP [21, 23]. Applying the Laplace transformation with respect to the variable $V$ to the partition function $Z(T, \mu, V)$ in the grand canonical ensemble, we obtain the isobaric partition function $\hat{Z}(T, \mu, z)$
[23]. The convenience of such a transformation consists in that the most right singularity of the isobaric partition function $z^{*}$ determines the pressure of the system $p=T z^{*}$. Just therefore, in order to study the phase structure of QGBM with surface tension, it is necessary to analyze the structure of singularities of $\hat{Z}(T, \mu, z)$ defined as follows:

$\hat{Z}(T, \mu, z) \equiv \int_{0}^{\infty} d V e^{-z V} Z(T, \mu, V)=\frac{1}{z-F(T, \mu, z)}$.

The function $F(T, \mu, z)=F_{H}(T, \mu, z)+F_{Q}(T, \mu, z)$ in the formula for $\hat{Z}(T, \mu, z)$ is the bulk spectrum of the model. Its discrete part

$F_{H}(z, T, \mu)=\sum_{j=1}^{n} g_{j} e^{\frac{b_{j} \mu}{T}-v_{j} z} \phi\left(T, m_{j}\right)$

corresponds to light hadrons with masses $m_{j}$, baryon charges $b_{j}$, own volumes $v_{j}$, degenerations $g_{j}$, and oneparticle densities

$\phi\left(T, m_{j}\right) \equiv \frac{1}{2 \pi^{2}} \int_{0}^{\infty} p^{2} d p e^{-\frac{\left(p^{2}+m_{j}^{2}\right)^{1 / 2}}{T}}$.

The large-volume bags in QGP are described by the continuous part of the bulk spectrum,

$F_{Q}(T, \mu, z)=u(T, \mu) I_{\tau}(\Delta z, \Sigma)$,

where we used the notation

$I_{\tau}(\Delta z, \Sigma) \equiv \int_{V_{0}}^{\infty} \frac{d v}{v^{\tau}} e^{-\Delta z v-\Sigma v^{\kappa}}$.

In addition, $\Delta z \equiv z-z_{Q}(T, \mu)$, and $T \Sigma$ stands for CST ( $\Sigma$ denotes the reduced CST). The effective surface area of a quark-gluon bag with volume $v$ is described in the continuous part of the spectrum of bags with the help of the parameter $\kappa$ as $v^{\kappa}$. As usual, $\kappa$ is determined by the space dimensionality $d$ as $\kappa=\frac{d-1}{d}$, but we interpret it here as a free parameter varying in the limits $0<\kappa<1$. The parameter $\tau$ is a Fisher exponent playing a significant role. It is worth noting that it takes different values in M1 $(1<\tau \leq 2)$ and M2 $(\tau>2)$. The model is solvable for a wide class of functions $u(T, \mu)$ and $z_{Q}(T, \mu)$, for which the only restrictions are the boundedness and the double differentiability for any values of arguments $[23,26,27]$. The meaning of the function $z_{Q}(T, \mu)$ consists in that it determines the pressure $p_{Q}$ inside a bag 


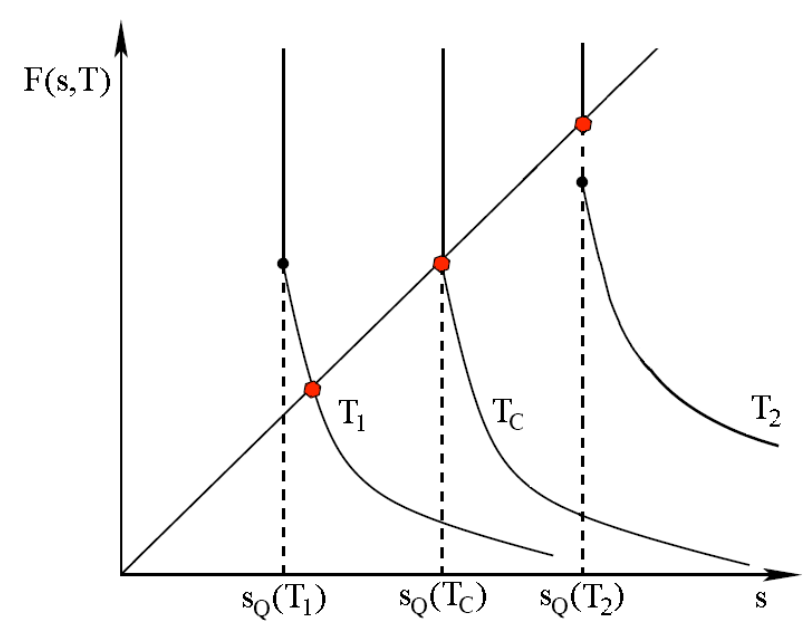

Fig. 1. Graphical solution of Eq. (6) in the PT region $\left(\mu>\mu_{\text {cep }}\right)$ in M1. The PT curve lies below the curve with the zero value of CST, i.e., $T_{c}(\mu)<T_{\Sigma}(\mu)$. Therefore, $F\left(z_{Q}, T, \mu\right)$ remains bounded for $T<T_{\Sigma}(\mu)$, where $\Sigma>0$. At small temperatures $T_{1}<T_{c}(\mu)$, the simple pole is the most right singularity of $\hat{Z}(T, \mu, z)$, and, therefore, the hadronic phase dominates. At higher temperatures $T_{c}(\mu)<T_{2}<T_{\Sigma}(\mu)$, the pressure in the system is determined by the essential singularity (7). Hence, the QGL phase dominates. At the critical temperature $T_{c}(\mu)$, there occurs PT, at which singularities (6) and (7) of the isobaric partition coincide

in QGP, namely, $p_{Q}=T z_{Q}(T, \mu)$. Therefore, in view of the data of lattice QCD, we may assert that $z_{Q}(T, \mu)$ is a monotonically increasing function of its arguments.

The very important specific feature of QGBM with surface tension is the dependence of the reduced CST $\Sigma(T, \mu)$ on the temperature $T$ and the baryon chemical potential $\mu$. We note that it becomes zero on the curve $T_{\Sigma}(\mu)$ of the phase diagram in the $\mu-T$ coordinates, i.e., $\Sigma\left(T_{\Sigma}, \mu\right)=0$. For a certain value of the baryon chemical potential $\mu$, the reduced CST is positive below this curve $\left(\Sigma\left(T<T_{\Sigma}, \mu\right)>0\right)$ and is negative above it $\left(\Sigma\left(T>T_{\Sigma}, \mu\right)<0\right)$. It is worth to note that, as was indicated in works $[23,26,27]$, the negative values of $\Sigma$ do not contradict the fundamental physical ideas. Indeed, CST in the grand canonical ensemble determines the surface free energy of a cluster $f_{\text {surf }}=e_{\text {surf }}-T s_{\text {surf }}$, where $e_{\text {surf }}$ and $s_{\text {surf }}$ are the surface energy and the entropy, respectively. Therefore, the inequality $\Sigma<0$ means only that the entropic contribution to the surface free energy exceeds that of the energy. This is a consequence of the appearance of such huge number of nonspherical deformations, which cannot be suppressed by the Boltzmann exponential factor. Just such behavior of the surface free energy (and, hence, CST) is foreseen by the exactly solvable model of surface deformations [29]. It is necessary to note that the conclusion on the possibility of

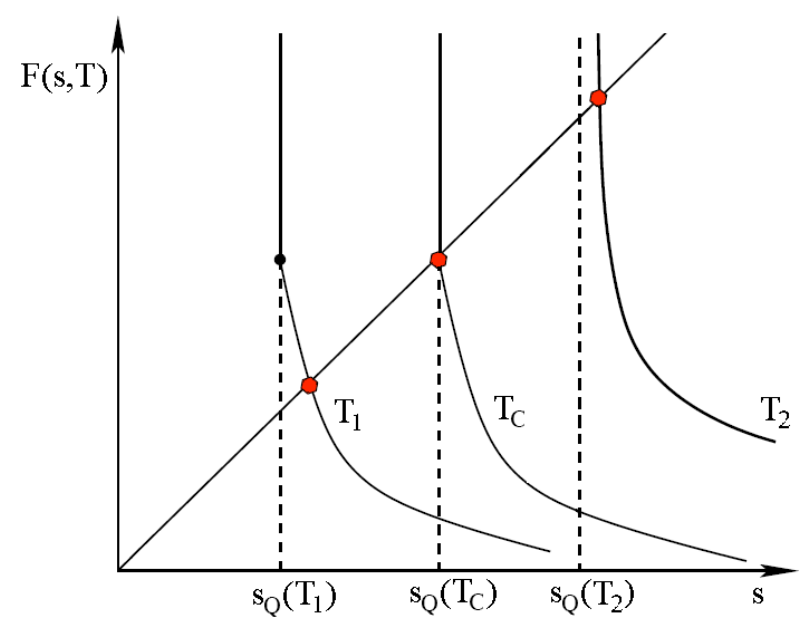

Fig. 2. Graphical solution of Eq. (6) in the PT region $(\mu>$ $\left.\mu_{\text {cep }}\right)$ in M2. The PT curve and the zero-CST curve coincide, i.e., $T_{c}(\mu)=T_{\Sigma}(\mu)$. Therefore, $F\left(z_{Q}, T, \mu\right)$ remains bounded for $T<T_{c}(\mu)$, where $\Sigma>0$. At small, $T_{1}<T_{c}(\mu)$, and large, $T_{2}>$ $T_{c}(\mu)$, temperatures, the simple pole is the most right singularity of $\hat{Z}(T, \mu, z)$. Therefore, the hadronic phase dominates. QGL exists only if singularities (6) and (7) of the isobaric partition coincide during $\mathrm{PT}$ at the temperature $T_{c}(\mu)$

negative values of CST was also made in works [30,31] on the basis of field-theoretic methods. Moreover, the recent consideration of the relation between the tension of confining color string and the surface tension of a bag in QGP $[32,33]$ has demonstrated that, in the cross-over region, $\Sigma$ is obligatorily negative.

As was noted above, the pressure in the system is determined by the most right singularity $z^{*}$ of the isobaric partition $\hat{Z}(T, \mu, z)$. The singularities are of two types: a simple pole, which corresponds to the gas phase and is defined by the equation

$z^{*}=F\left(T, \mu, z^{*}\right)$

and the essential singularity, which corresponds to the liquid phase and is given by

$z^{*}=z_{Q}(T, \mu)$

By graphically solving the equation $z=F(T, \mu, z)$, we can determine the most right singularity from (6) and (7) [26]. Let us make it for the simplest case of the constant baryon chemical potential. First, we consider the region of PT $\mu>\mu_{\text {cep }}$ (Fig. 1 in the case of M1 and Fig. 2 in the case of M2). CST is positive in this region at temperatures less than $T_{\Sigma}(\mu)$. Hence, integral (5) together with the model spectrum $F(z, T, \mu)$ remain bounded for $z \geq z_{Q}(T, \mu)$. Therefore, for $T_{1}<T_{\Sigma}(\mu)$, the singularity defined by Eq. (6) is located to the right from $z_{Q}\left(T_{1}, \mu\right)$, 


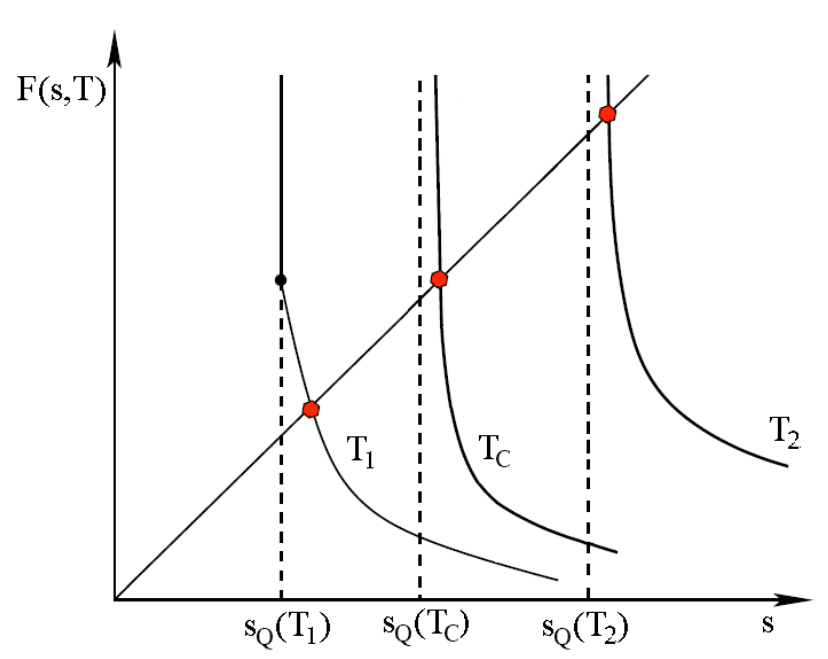

Fig. 3. Graphical solution of Eq. (6) in the cross-over region $(\mu \leq$ $\left.\mu_{\text {cep }}\right)$, where CST is negative [23, 26], and, therefore, $F\left(z_{Q}, T, \mu\right)$ remains bounded only at small temperatures. Then the simple pole (6) is always the most right singularity of the isobaric partition

and the system exists in the hadron gas phase. We note that the difference $z^{*}-z_{Q}(T, \mu)$ decreases, as the temperature increases. Therefore, at its some critical value $T_{c}(\mu) \leq T_{\Sigma}(\mu)$, singularities (6) and (7) become identical, i.e., $z_{Q}\left(T_{c}, \mu\right)=F\left(T_{c}, \mu, z_{Q}\left(T_{c}, \mu\right)\right)$. It is worth noting that since the pressure in the system is determined by the most right singularity of the isobaric partition function as $p=z^{*} T$, this condition is just the Gibbs criterion of phase equilibrium [23, 26, 27]. Hence, the curve $T_{c}(\mu)$ should be identified with the deconfinement PT curve. Moreover, at temperatures $T_{2} \in\left(T_{c}(\mu), T_{\Sigma}(\mu)\right)$, the most right singularity $\hat{Z}(T, \mu, z)$ is an essential one. Therefore, the system exists in the state of a quark-gluon liquid (QGL) undergone the deconfinement. Along the curve with the zero value of $\operatorname{CST} T_{\Sigma}(\mu)$, singularities (6) and (7) become again identical. Hence, there occurs a surface-induced PT of the second or higher order [26]. We note that it exists only in M1. In M2, this phase transition coincides with the deconfinement $\mathrm{PT}$, because $T_{c}(\mu)=T_{\Sigma}(\mu)$ in this model. In addition, the true QGL, whose pressure is determined by the essential singularity (7) in M2, exists only along the curve of coexistence of phases, $T_{c}(\mu)$. These two circumstances are basic for the difference in the phase structures of M1 and M2. As the temperature further increases, CST becomes negative, and $F(T, \mu, z)$ becomes divergent for $z \leq z_{Q}(T, \mu)$. Hence, at temperatures higher than $T_{\Sigma}(\mu)$, the most right singularity of the isobaric partition function is the simple pole (6), which corresponds to QGP in the deconfined gas phase. The above analysis concerns

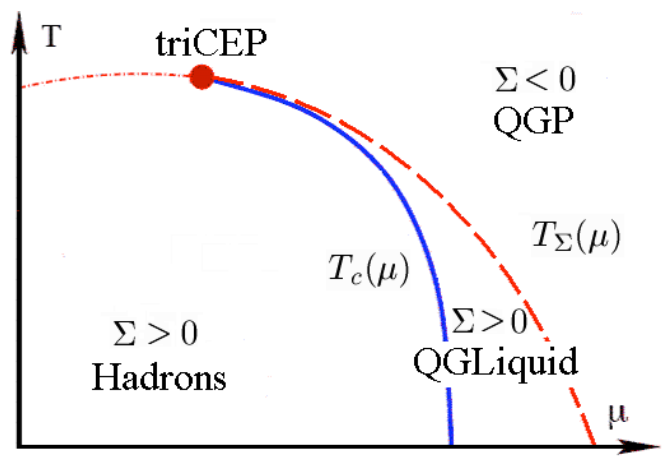

Fig. 4. Schematic representation of the phase diagram in M1 in the $\mu-T$ coordinates. CST changes the sign along the dotted curve $T_{\Sigma}(\mu)$. The deconfinement PT occurs along the continuous curve $T_{c}(\mu)$ for $\mu>\mu_{\mathrm{cep}}$. For $\mu<\mu_{\mathrm{cep}}$, it degenerates in the cross-over (dash-dotted curve $\left.T_{\Sigma}(\mu)\right)$. These regions are separated by TCP, which is marked by a circle

the region $\mu>\mu_{\text {cep }}$ of the phase diagram, where the first order deconfinement PT exists. For $\mu<\mu_{\text {cep }}$, PT degenerates in the continuous cross-over transition (Fig. 3). In this case, the type of the most right singularity of the isobaric distribution is not changed, since $\Sigma$ is negative in this region $[23,26]$.

In the grand canonical ensemble, the pressures in the liquid (QGL) and gas (hadrons or QGP) phases of the given model are, respectively,

$p_{L}(T, \mu)=T z_{Q}(T, \mu)$,

$p_{G}(T, \mu)=T\left[F_{H}(z, T, \mu)+u(T, \mu) I_{\tau}(\Delta z, \Sigma)\right]$.

Moreover, the pressure in the hadronic phase $p_{H} \equiv$ $\left.p_{G}\right|_{T<T_{C}}$, and $\left.p_{\mathrm{QGP}} \equiv p_{G}\right|_{T>T_{\Sigma}}$ (in M2, $T_{\Sigma}=T_{c}$ ) is the pressure in QGP. It is convenient to denote the quantities defined at the temperatures larger or smaller than $T_{c}$ by the indices - and + . Then, for example, $p_{T=T_{c} \pm 0} \equiv p^{ \pm}$.

\subsection{Model with tricritical point}

M1 was exactly solved in $[23,26]$. Its phase structure includes the hadronic phase and the phases of QGP and QGL. The phase diagram of M1 in the $\mu-T$ coordinates is given in Fig. 4. In this model, $p^{-}=p_{H}$ and $p^{+}=$ $p_{Q G L}$. The deconfinement $\mathrm{PT}$ from the hadronic phase to the QGL phase occurs for $\mu>\mu_{\text {cep }}$ along the curve $T_{c}(\mu)$, which is positioned below the curve with the zero value of $\operatorname{CST} T_{\Sigma}(\mu)$. For $\mu<\mu_{\text {cep }}$, PT degenerates in the cross-over. These two regions are separated by TCP with the coordinates $\left(\mu_{\mathrm{cep}}, T_{\mathrm{cep}}\right)$. The necessary condition for the existence of a deconfinement $\mathrm{PT}$ of the first order and its TCP in M1 is $\frac{3}{2}<\tau \leq 2$ [23]. 
A new parametrization of the reduced CST was first proposed in [23]. However, it is worth to note that, in the present work, it will be studied in the more general case,

$\Sigma(T, \mu)=\operatorname{sign}\left(T-T_{\Sigma}(\mu)\right) \frac{\sigma}{T}\left|\frac{T_{\Sigma}(\mu)-T}{T_{\Sigma}(\mu)}\right|^{\zeta}$,

which admits any positive values of parameter $\zeta$ for the validity of the condition $\Sigma\left(T_{\Sigma}\right)=0$. The parameter $\sigma$ is some positive constant, which has the meaning of CST at the temperature $T=0$. However, it can be interpreted also as a smooth regular function of the temperature and/or the baryon chemical potential, which has no influence on the results.

In a neighborhood of TCP, the behavior of the deconfinement PT curve and the zero-CST curve in the $\mu-T$ coordinates can be described by one parameter $\xi^{T}>0$ :

$T_{\text {cep }}-T_{\Sigma}(\mu) \sim T_{\text {cep }}-T_{c}(\mu) \sim\left(\mu-\mu_{\text {cep }}\right)^{\xi^{T}}$.

This is a consequence of the fact that the curves $T_{c}(\mu)$ and $T_{\Sigma}(\mu)$ do not intersect each other, but touch at TCP. The proof of this assertion and the analysis of the conditions sufficient for its validity will be presented below. This parameter allows us to describe the phase diagram of M1 in the most general way. Its extraordinary meaning consists in that M1 can describe the critical exponents of the three-dimensional Ising model, simple liquids, $\mathrm{O}(4)$-symmetric three-dimensional spin model, etc. only due to it. For example, it is impossible for MSMF [34]. In addition, the parameter $\xi^{T}$ is uniquely connected with the quantity $K_{c}$, which was introduced in $[10,11]$ and plays the crucial role in the classification of the types of critical points.

Along the curve of phase equilibrium, the ClapeyronClausius equation can be written in the form $\frac{d \mu_{c}}{d T}=$ $-\left.\frac{s^{-}-s^{+}}{\rho^{-}-\rho^{+}}\right|_{T=T_{c}}$. The baryon density $\rho$ and the entropy density $s$ of each phase are determined as the partial derivatives of the pressure of the given phase with respect to the baryon chemical potential $\mu$ and the temperature $T$. With the help of (8) and (9), this equation can be written explicitly as

$$
\frac{d \mu_{c}}{d T}=-\left.\frac{A_{T}-\frac{\partial \Sigma}{\partial T} u I_{\tau-\kappa}(0, \Sigma)}{A_{\mu}-\frac{\partial \Sigma}{\partial \mu} u I_{\tau-\kappa}(0, \Sigma)}\right|_{T=T_{c}},
$$

where

$$
A_{i} \equiv \frac{\partial F_{H}}{\partial i}+\frac{\partial u}{\partial i} I_{\tau}+\frac{\partial z_{Q}}{\partial i}\left(\frac{\partial F_{H}}{\partial z}-1\right)
$$

for $i \in\{T, \mu\}$. The parametrization of the reduced CST (10) and the shape of the curve $T_{c}(\mu)(11)$ together with (12) allow us to conclude that

$$
\begin{aligned}
& \left.A_{T}\right|_{T=T_{c}} \sim\left(T_{\text {cep }}-T_{c}(\mu)\right)^{\chi+\frac{1}{\xi^{T}}-1}, \\
& \left.A_{\mu}\right|_{T=T_{c}} \sim\left(T_{\text {cep }}-T_{c}(\mu)\right)^{\chi},
\end{aligned}
$$

where the exponent $\chi$ was first introduced in [35]. Its appearance is a direct consequence of the ClapeyronClausius equation which naturally follows from the Gibbs criterion of phase equilibrium. Thus, the introduction of the parameter $\chi$ is quite general and is valid for any model with PT of the liquid-vapor type. However, this exponent was not used yet in the analysis of critical exponents in other models. For example, this parameter was implicitly considered to be zero in MSMF [34]. The possible values of this parameter are determined by the inequality $\chi \geq \max \left(0,1-\frac{1}{\xi^{T}}\right)$ due to the boundedness of the integral $I_{\tau}$ and the functions $F_{H}, u$, and $z_{Q}$ together with their first derivatives for any values of $T$ and $\mu$.

Let us turn to the question about the behavior of the curves $T_{c}(\mu)$ and $T_{\Sigma}(\mu)$ in a neighborhood of TCP, as well as the reduced $\operatorname{CST} \Sigma=0$ and the parameter $\Delta z=$ 0 along the curve $T_{\Sigma}(\mu)$. Therefore, an analog of the Clapeyron-Clausius equation (12) for this curve takes the form $\frac{d \mu_{\Sigma}}{d T}=-\left.\frac{A_{T}}{A_{\mu}}\right|_{T=T_{\Sigma}}$. If the functions $A_{T}$ and $A_{\mu}$ satisfy the quite general requirements of analyticity, we may assert that such a relation between them holds to within some insignificant factor $f$ for any curve lying in a neighborhood of TCP. In other words,

$A_{T}=-f A_{\mu} \frac{d \mu_{\Sigma}}{d T}$

in a neighborhood of TCP. It is worth noting that $f$ must be a smooth function of $T$ and/or $\mu$ and have no singularities or even be a constant. Basing on the relation $\left(T_{c}(\mu)-T_{\Sigma}(\mu)\right)_{\mu \rightarrow \mu_{\text {cep }}} \rightarrow 0$ and using the parametrization of the reduced CST (10), we obtain

$\left.\frac{\partial \Sigma}{\partial T}\right|_{T=T_{c}} \simeq-\left.\frac{T_{\Sigma}}{T_{c}} \frac{d \mu_{\Sigma}}{d T} \frac{\partial \Sigma}{\partial \mu}\right|_{T=T_{c}}$.

This result together with Eqs. (12) and (16) allow us to assert that $\frac{d \mu_{c}}{d T}=\frac{d \mu_{\Sigma}}{d T}$ at TCP. This is possible only under the condition that the curves $T_{c}(\mu)$ and $T_{\Sigma}(\mu)$ do not intersect each other, but touch at TCP. Hence, their behavior can be described, as above, by one parameter $\xi^{T}$. It is worth noting that, in this case, the functions $A_{T}$ and $A_{\mu}$ must satisfy the quite general requirements of analyticity in a neighborhood of TCP. 


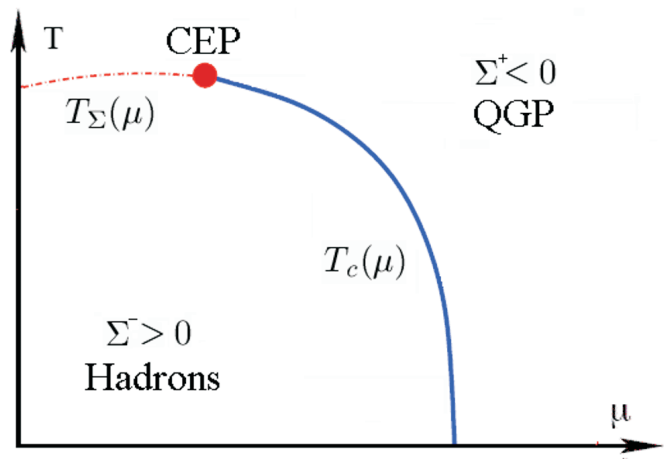

Fig. 5. Schematic representation of the phase diagram of M2 in the $\mu-T$ coordinates. The deconfinement PT occurs along the continuous curve $T_{c}(\mu)$ for $\mu>\mu_{\text {cep }}$. For $\mu<\mu_{\text {cep }}$, it degenerates in the cross-over (dotted curve $T_{\Sigma}(\mu)$ ). For $\mu$ larger than the critical value $\mu_{\mathrm{cep}}$, CST changes the sign exactly on the phase equilibrium curve, i.e., $T_{\Sigma}(\mu)=T_{c}(\mu)$ for $\mu>\mu_{\text {cep }}$. The regions of PT and cross-over are separated by $\mathrm{CP}$, which is marked with a circle

\subsection{Model with critical point}

The exact solution in M2 was found in [27]. Like the case of M1, the phase structure of M2 includes the hadronic phase and the phases of QGP and QGL. The main difference of the phase diagram of M2 from that of M1 consists in that CST changes the sign exactly on the curve of phase equilibrium for the baryon chemical potentials larger than $\mu_{\text {cep }}$, i.e., $T_{\Sigma}(\mu)=T_{c}(\mu)$ for $\mu>\mu_{\text {cep }}$. Thus, as was mentioned above, the surface-induced PT of the second order in M2 "is hidden" inside of a deconfinement PT of the first order. As a result, the true QGL exists only along the phase equilibrium curve $T_{c}(\mu)$. The schematic representation of the phase diagram of M2 in the coordinates $(\mu-T)$ is presented in Fig. 5. We note that, in this model, $p^{-}=p_{H}$ and $p^{+}=p_{\mathrm{QGP}}$. In [27], it was demonstrated that a deconfinement $\mathrm{PT}$ of the first order and its CP exist in M2 for $\tau>2$.

The parametrization of the reduced CST in M2 is similar to that in M1. However, it differs from (10) by that the parameter

$\sigma^{ \pm}(T, \mu)=\sigma_{0}^{ \pm}\left(T-T_{\text {cep }}+\frac{d T_{c}}{d \mu}\left(\mu_{\text {cep }}-\mu\right)\right)^{\xi^{ \pm}}$

is not a constant. In addition, the values of constant parameters $\sigma_{0}^{ \pm}, \zeta^{ \pm}$, and $\xi^{ \pm}$in the general case are different above and below the phase equilibrium curve. This follows from the fact that PT of the first order in M2 is generated due to the discontinuity of partial derivatives of the reduced CST at the passage across the curve $T_{c}(\mu)$ [27]. It is worth noting that the requirement for the jump of the baryon density to be positive at the deconfinement PT, i.e., $\rho^{+}\left(T_{c}\right)>\rho^{-}\left(T_{c}\right)$, can be satisfied only for $\zeta^{+}=1$.

The behavior of the PT curve in a neighborhood of CP, like that in M1, is parametrized with the parameter $\xi^{T}$ with the help of Eq. (11), i.e., $T_{\text {cep }}-T_{c}(\mu) \sim\left(\mu-\mu_{\text {cep }}\right)^{\xi^{T}}$. The analysis of the Clapeyron-Clausius equation, which holds along the curve $T_{c}(\mu)$, allows us to describe the behavior of the functions $A_{T}$ and $A_{\mu}$ like that in M1 in terms of the parameter $\chi$ (see (14) and (15)).

\section{Critical Exponents}

Traditionally, the behavior of a system in a neighborhood of the critical point is described by the critical exponents $\alpha, \alpha^{\prime}, \beta, \gamma^{\prime}$, and $\delta[3-5]$. For example, the heat capacity of the system, $\left.C_{\rho} \equiv \frac{T}{\rho} \frac{d s}{d T}\right|_{\rho}$, along the critical isochore $\rho=\rho_{\text {cep }}$ takes the form

$C_{\rho} \sim\left\{\begin{aligned}|t|^{-\alpha^{\prime}}, & \text { for } t \leq 0 \\ t^{-\alpha}, & \text { for } t>0\end{aligned}\right.$

the jump of the baryon density $\Delta \rho \equiv\left(\rho^{+}-\rho^{-}\right)_{T=T_{c}}$ vanishes, while approaching the critical point, by the law

$\Delta \rho \sim|t|^{\beta}, t \leq 0$,

and the jump of the isothermal compressibilities $K_{T}=$ $\left.\frac{1}{\rho} \frac{d \rho}{d p}\right|_{T}$ diverges at the critical point as

$\Delta K_{T} \sim|t|^{-\gamma^{\prime}}, t \leq 0$,

where $\Delta K_{T} \equiv\left(K_{T}^{+}-K_{T}^{-}\right)_{T=T_{c}}$. Here, the variable $t \equiv \frac{T-T_{\mathrm{cep}}}{T_{\mathrm{cep}}}$ means the reduced temperature. The shape of the critical isotherm $T=T_{\text {cep }}$ is determined by the exponent $\delta$ :

$p_{\text {cep }}-\widetilde{p} \sim\left(\rho_{\text {cep }}-\widetilde{\rho}\right)^{\delta}$,

where the tilde marks the quantities defined at $t=0$.

In order to determine the critical exponent $\alpha^{\prime}$, it is necessary to find the total derivative of the entropy density along the critical isochore located inside the mixed phase with respect to the temperature [2-5]. At fixed values of $T$ and $\mu$, the state of the system on this curve is determined by the volume fraction of the hadronic phase $\lambda$ :

$\left.\rho\right|_{T=T_{c}}=\left.\lambda \rho^{-}\right|_{T=T_{c}}+\left.(1-\lambda) \rho^{+}\right|_{T=T_{c}}$,

$\left.s\right|_{T=T_{c}}=\left.\lambda s^{-}\right|_{T=T_{c}}+\left.(1-\lambda) s^{+}\right|_{T=T_{c}}$. 
It is worth noting that $1-\lambda$ is the volume fraction of QGL in M1 or QGP in M2. Using these equations, we can obtain the expression for $\frac{d s}{d T}$ along the critical isochore. Together with the Clapeyron-Clausius equation, it yields

$C_{\rho_{\mathrm{cep}}}=\frac{T_{c}}{\rho_{\text {cep }}}\left[\left(\rho_{Q}-\rho_{\mathrm{cep}}\right) \frac{d^{2} \mu_{c}}{d T^{2}}+\frac{d s_{Q}}{d T}+\frac{d \mu_{c}}{d T} \frac{d \rho_{Q}}{d T}\right]_{T=T_{c}}$,

where the index $Q$ marks, here and below, all quantities related to QGL. For some universality classes (e.g., for three-dimensional $\mathrm{O}(4)$-symmetric spin systems), the exponent $\alpha^{\prime}$ is negative. Therefore, in order to attain a higher generality of the present model, it is of importance to ensure the generation of $\alpha^{\prime}<0$ in it. According to definition (19), this occurs if $C_{\rho}$ is equal to zero at the critical point. Expanding the difference $\rho_{Q}-\rho_{\text {cep }}$ in a series of deviations of the temperature and the chemical potential from the critical values and using (11), it is possible to show that the first term in (25) behaves as $|t|^{\min \left(1, \frac{1}{\xi^{T}}\right)+\frac{1}{\xi^{T}}-2}$. We also note that the baryon density of QGL $\rho_{Q}$ is defined as the derivative of $p_{Q}=T z_{Q}(T, \mu)$ with respect to the baryon chemical potential $\mu$. Thus, by virtue of the boundedness and the double differentiability of $p_{Q}$, the behavior of the third term in (25) is determined by the squared derivative $\frac{d \mu_{c}}{d T}$, i.e., by $|t| \frac{2}{\xi^{T}}-2$. Hence, for $\frac{1}{\xi^{T}}>1$, the first and third terms in (25) are equal to zero at the critical point. Thus, the exponent $\alpha^{\prime}$ in the present model can be negative only if $\xi^{T}<1$ and $\left.\frac{d s_{Q}}{d T}\right|_{\text {cep }}=0$. The last relation holds for

$$
\left.\frac{\partial s_{Q}}{\partial T}\right|_{T=T_{c}} \sim|t|^{\omega}
$$

where the parameter $\omega \geq 0$. Moreover, the relation $\omega=0$ corresponds to the derivative $\frac{\partial s_{Q}}{\partial T}$, which is bounded at CP. For $\omega>0$, this derivative is equal to zero. Geometrically, this means that, for positive $\omega$, the function $s_{Q}\left(T, \mu_{\text {cep }}\right)$ has a deflection at the critical temperature. Relation (26) implies that the second term in (25) behaves as $|t|^{\min \left(\omega, \frac{1}{\xi^{T}}-1\right)}$. Hence, in view of definition (19), we have

$\alpha^{\prime}=2-2 \min \left(\frac{1}{\xi^{T}}, 1+\frac{\omega}{2}, \frac{1+\frac{1}{\xi^{T}}}{2}\right)$.

The formula for the critical exponent $\alpha^{\prime}$ allows us to show that it is nonnegative for $\omega=0$, whereas $\left.\alpha^{\prime}\right|_{\omega>0}<$ 0 for $\xi^{T}<1$. This yields the result, which is extremely important for the analysis of the values of critical exponents. Namely, $\alpha^{\prime}<0$ if the derivative $\left.\frac{\partial s_{Q}}{\partial T}\right|_{\text {cep }}$ equals zero, and its finite values correspond to the nonnegative values of this exponent. It is worth noting that the formulas for $\alpha^{\prime}$ are completely identical in M1 and M2 and include only the parameters $\xi^{T}$ and $\omega$. On the physical basis, this can be explained by that the given exponent describes the heat capacity of the system along the critical isochore. In both versions of QGBM with surface tension, this isochore lies completely inside the mixed phase, and its shape in the plane $\mu-T$ is described by the parameter $\xi^{T}$.

In both versions of QGBM with surface tension, the system exists in the state of QGP at temperatures higher than the critical one. Therefore, in order to determine the critical exponent $\alpha$, it is necessary to find the total derivative of the entropy $s^{+}$with respect to the temperature along the critical isochore $\rho^{+}=\rho_{\text {cep }}$. Then, by using the standard notations of the baryon density and the entropy, we obtain with regard for Eq. (9) that, for $T \geq T_{\text {cep }}$,

$C_{\rho}=\frac{T}{\rho_{\text {cep }}} \frac{d}{d T}\left[\Delta z+s_{Q}+\left(\rho_{\text {cep }}-\rho_{Q}\right) \frac{A_{T}-\frac{\partial \Sigma}{\partial T} u I_{\tau-\kappa}}{A_{\mu}-\frac{\partial \Sigma}{\partial \mu} u I_{\tau-\kappa}}\right]_{\rho_{\text {cep }}}$

Due to the analyticity of the function $z_{Q}$ for any finite values of its arguments, we may assert that parametrization (26) holds not only on the PT curve, but also in the whole neighborhood of the critical point. This implies that the second term in $(28)$ behaves as $|t|^{\min \left(\omega, \frac{1}{\xi^{T}}-1\right)}$. In addition, using the parametrization of CST and Eq. (16) and expanding the difference $\rho_{\text {cep }}-\rho_{Q}$ up to the terms linear in the deviations of the temperature and the chemical potential from the critical values, we obtain that the behavior of the third term in (28) is described by the formula $t^{\min \left(\frac{1}{\xi^{T}}-1, \frac{2}{\xi^{T}}-2\right)}$. Hence, in order to find $\alpha$, it is necessary to analyze the behavior of $\Delta z$, which is defined in a neighborhood of the critical point as

$$
\Delta z=\frac{\left.A_{T}\right|_{\mathrm{cep}} \Delta T+\left.A_{\mu}\right|_{\mathrm{cep}} \Delta \mu-\Sigma u I_{\tau-\kappa}}{1-\frac{\partial F_{H}}{\partial s}+u I_{\tau-1}} .
$$

We note that the difference in the behavior of this quantity in M1 and M2 leads to the formulas for the critical exponent $\alpha$, which are different in the general case in these models.

The deviations of the densities of pure phases from the critical value tend to zero, while approaching TCP or CP. Therefore, it is convenient to introduce the exponents $\beta^{+}$and $\beta^{-}$, which describe their behavior, i.e.,

$\left(\rho^{ \pm}-\rho_{\text {cep }}\right)_{T=T_{c}} \sim|t|^{\beta^{ \pm}}$. 
In terms of these exponents, we have

$\beta=\min \left(\beta^{+}, \beta^{-}\right)$.

It is worth noting that the condition of boundedness of the volume fraction of the low-temperature phase $\lambda$ and the volume fraction of the high-temperature phase $1-\lambda$ at $\mathrm{CP}$ in $\mathrm{M} 2$ yields the relation $\beta<\min \left(1, \frac{1}{\xi^{T}}, \chi\right)$ [35]. In $\mathrm{M} 1$, its analog is $\beta<\min \left(1, \frac{1}{\xi^{T}}\right)$. Hence, in order to determine $\beta$, it is necessary to study the behavior of the deviations of the densities of pure phases from $\rho_{\text {cep }}$. For the liquid phase, such as QGL, the expansion in a series up to the terms linear in the deviations of $T$ and $\mu$ from the critical values gives

$\left.\left(\rho_{L}-\rho_{\mathrm{cep}}\right)_{T=T_{c}} \simeq \Delta T \frac{\partial \rho_{Q}}{\partial T}\right|_{\text {cep }}+\left.\Delta \mu \frac{\partial \rho_{Q}}{\partial \mu}\right|_{\text {cep }}$.

In the same way, we can show that, for the gas phase including hadrons and QGP, a deviation of the density from the critical value reads

$$
\begin{aligned}
& \left(\rho_{G}-\rho_{\mathrm{cep}}\right)_{T=T_{c}} \simeq \\
& \simeq\left[T \frac{A_{\mu}-\frac{\partial \Sigma}{\partial \mu} u I_{\tau-\kappa}}{1-\frac{\partial F_{H}}{\partial z}+u I_{\tau-1}}+\rho_{L}-\rho_{\mathrm{cep}}\right]_{T=T_{c}} .
\end{aligned}
$$

Formulas (32), (33), (11), and (15) and the parametrization of CST allow us to determine $\beta^{+}$and $\beta^{-}$and, hence, the critical exponent $\beta$.

In order to find the critical exponent $\gamma^{\prime}$, it is convenient to represent the derivative $\left.\frac{d \rho}{d p}\right|_{T}$ as the ratio of the relevant differentials at a constant temperature. Then, using the definition of the baryon density $\rho=\frac{\partial p}{\partial \mu}$, we finally obtain

$\Delta K_{T}=\left[\frac{1}{\rho^{+^{2}}} \frac{\partial^{2} p^{+}}{\partial \mu^{2}}-\frac{1}{\rho^{-2}} \frac{\partial^{2} p^{-}}{\partial \mu^{2}}\right]_{T=T_{c}}$.

Relations (8) and (9) yield

$\frac{\partial^{2} p_{L}}{\partial \mu^{2}}=T \frac{\partial^{2} z_{Q}}{\partial \mu^{2}}$

for the liquid phase and

$\frac{\partial^{2} p_{g}}{\partial \mu^{2}} \simeq T \frac{\left(\frac{\partial \Delta z}{\partial \mu}\right)^{2} u I_{\tau-2}-\frac{\partial^{2} \Sigma}{\partial \mu^{2}} u I_{\tau-\kappa}}{1-\frac{\partial F_{H}}{\partial z}+u I_{\tau-1}}$

for the gas phase. We note that relation (36) includes only the terms, which are most singular at TCP or CP.
In addition, the first term in the previous formula is principal in M1, whereas the second term dominates in M2. Just this leads to the difference of the formulas for the exponent $\gamma^{\prime}$ in both models.

The zero-CST curve is convex in the $T-\mu$ plane both in M1 and M2 (see Figs. 4 and 5). Therefore, in both models, the critical isotherm is located in the region, where $\Sigma$ is negative everywhere, except for TCP or CP. As a result, in both versions of QGBM with surface tension for $T=T_{\text {cep }}$ and $\mu \neq \mu_{\text {cep }}$, the system exists in the state of QGP, which is a gas phase with the pressure determined by Eq. (9). Therefore, expanding the deviations of the pressure and the density of QGP from the critical values up to the terms linear in $\Delta \mu=\mu-\mu_{\text {cep }}$, we obtain

$\tilde{p}-p_{\text {cep }}=\Delta \mu \rho_{\text {cep }}$

$\tilde{\rho}-\rho_{\text {cep }}=T_{\text {cep }} \frac{\partial \tilde{\Delta}_{z}}{\partial \mu}$

where the definitions of the baryon density $\rho=\frac{\partial p}{\partial \mu}$ and the variable $\Delta z$ were used. We note that the full formula for the deviation of the density of QGP from the critical value includes the term $\left.T_{\text {cep }} \Delta \mu \frac{\partial^{2} z_{Q}}{\partial \mu^{2}}\right|_{\text {cep }}$. However, the imposition of the condition $\delta>1$ makes it insignificantly small, which gives (38). Thus, by analyzing the behavior of

$$
\frac{\partial \tilde{\Delta}_{z}}{\partial \mu}=\left.\frac{A_{\mu}-\frac{\partial \Sigma}{\partial \mu} u I_{\tau-\kappa}}{1-\frac{\partial F_{H}}{\partial z}+u I_{\tau-1}}\right|_{T=T_{\mathrm{cep}}}
$$

we can determine the critical exponent $\delta$.

\subsection{Critical exponents of M1}

At the supercritical temperatures, the shape of the isochore $\rho=\rho_{\text {cep }}$ is determined by the derivative $\frac{d \mu_{\rho_{\text {cep }}}}{d T}=$ $-\frac{\partial \rho}{\partial T} / \frac{\partial \rho}{\partial \mu}$. Considering only the principal terms in the derivatives of the baryon density, we can show that, in a neighborhood of TCP, $\frac{d \mu_{\rho_{\mathrm{cep}}}}{d T} \simeq-\frac{\partial \Delta z}{\partial T} / \frac{\partial \Delta z}{\partial \mu} \sim \frac{d \mu_{\Sigma}}{d T}$. Hence, the shape of the critical isochore is also determined by the parameter $\xi^{T}$. Then, using (28) and (29), we obtain that

$\left.\alpha\right|_{\chi \neq 1-\frac{1}{\xi^{T}}}=\alpha^{\prime}$

$\left.\alpha\right|_{\chi=1-\frac{1}{\xi^{T}}}=\max \left[\alpha^{\prime}, \frac{\frac{\zeta}{\kappa} \min (\kappa, \tau-1)}{\tau-1}-1\right]$, 
in M1. Using (32) and (33) together with the parametrization accepted in $\mathrm{M} 1$, we can show that

$\beta^{+}=\min \left(1, \frac{1}{\xi^{T}}\right)$

$\beta^{-}=\frac{\zeta}{\kappa}(2-\tau)+\min \left[\chi, \frac{\zeta}{\kappa} \min (\kappa, \tau-1)-\frac{1}{\xi^{T}}\right]$

in the present model. The above-presented condition $\beta<\min \left(1, \frac{1}{\xi^{T}}\right)$ yields $\beta=\beta^{-}$in M1. The other critical exponents of this model are as follows:

$\gamma^{\prime}=\frac{\zeta}{\kappa}-2 \beta$

$\left.\delta\right|_{\chi=0}=\left[\frac{\min \left[\xi^{T} \frac{\zeta}{\kappa} \min (\tau-1, \kappa), 1\right]}{\tau-1}-1\right]^{-1}$

$\left.\delta\right|_{\chi>0}=\left[\frac{\xi^{T} \frac{\zeta}{\kappa} \min (\tau-1, \kappa)}{\tau-1}-1\right]^{-1}$.

As is seen from formulas (27) and (40)-(46), the spectrum of the values of critical exponents of model M1 is quite wide. In this model, we can obtain both positive and negative values of exponent $\alpha^{\prime}$, all positive values of exponents $\beta$ and $\gamma^{\prime}$, and the value of exponent $\delta$, which is more than 1 . This testifies to a significant degree of generality of the model, which allows one to describe the critical exponents of the twoand three-dimensional Ising models, simple liquids, and three-dimensional $\mathrm{O}(2)-, \mathrm{O}(3)$-, and $\mathrm{O}(4)$-symmetric spin models, which are presented in Table 1 . The values of model parameters, which generate the critical exponents of the indicated physical systems and models, are given in Table 2.

Since model M1 is formulated for $1<\tau \leq 2$, and this parameter takes the same values in MSMF [24], it is of

T a b l e 1. Critical exponents of the two-dimensional Ising model [4], simple liquids [4], three-dimensional Ising model [9], three-dimensional $\mathrm{O}(2)-[36], \mathrm{O}(3)-[37]$, and $\mathrm{O}(4)$-symmetric $[38,39]$ spin models

\begin{tabular}{c|c|c|c|c|c|c}
\hline & $\begin{array}{c}\text { Simple } \\
\text { liquids }\end{array}$ & $\begin{array}{c}2 \mathrm{D} \\
\text { Ising }\end{array}$ & $\begin{array}{c}3 \mathrm{D} \\
\text { Ising }\end{array}$ & $\begin{array}{c}\mathrm{O}(2) \\
\text { model }\end{array}$ & $\begin{array}{c}\mathrm{O}(3) \\
\text { model }\end{array}$ & $\begin{array}{c}\mathrm{O}(4) \\
\text { model }\end{array}$ \\
\hline$\alpha^{\prime}$ & $0.10(1)$ & 0 & $0.1096(5)$ & $-0.0146(8)$ & $-0.1336(15)$ & $-0.19(6)$ \\
$\beta$ & $0.335(15)$ & $1 / 8$ & $0.3265(1)$ & $0.3485(2)$ & $0.3689(3)$ & $0.38(1)$ \\
$\gamma^{\prime}$ & $1.25(5)$ & $7 / 4$ & $1.2373(2)$ & $1.3177(5)$ & $1.3960(9)$ & $1.44(4)$ \\
$\delta$ & $4.5(3)$ & 15 & $4.7893(8)$ & $4.780(2)$ & $4.78(3)$ & $4.82(5)$ \\
\hline
\end{tabular}

interest to analyze the interrelation of the critical exponents in these models. The importance of such a comparison is related to the fact that the end point of $\mathrm{PT}$ is namely TCP both in M1 and MSMF. In other words, the phase diagrams of these models are similar to each other to a significant degree. The explicit formulas for the critical exponents of the present model show that it realizes a mode, where the critical exponents in M1 reproduce the critical exponents of MSMF $\alpha_{\mathrm{MSMF}}^{\prime}=0, \beta_{\mathrm{MSMF}}=$ $\frac{\zeta}{\kappa}(2-\tau), \gamma_{\mathrm{MSMF}}^{\prime}=\frac{\zeta}{\kappa}(2 \tau-3)$, and $\delta_{\mathrm{MSMF}}=\frac{\tau-1}{2-\tau}[34]$. Indeed, this occurs for $\frac{\zeta}{\kappa} \min (\kappa, \tau-1)-\frac{1}{\xi^{T}} \geq \chi=0$ and $\frac{1}{\xi^{T}} \geq 1$. This fact testifies clearly that M1 is more general, than MSMF. Therefore, the former includes the solutions of the latter as a partial case. It is worth noting that, for $\xi^{T}>1$ and $\frac{\zeta}{\kappa} \min (\kappa, \tau-1)-\frac{1}{\xi^{T}} \geq \chi=0$, model M1 can generate a mode, where the exponents $\beta, \gamma^{\prime}$, and $\delta$ coincide with the appropriate exponents of MSMF, whereas $\alpha^{\prime}$ is positive and depends only on the parameter $\xi^{T}$. Just this circumstance allows model M1, as distinct from MSMF, to describe the critical exponents of the three-dimensional Ising model and simple liquids.

One more extremely interesting mode in M1 is realized for $\chi \geq \frac{\zeta}{\kappa} \min (\kappa, \tau-1)-\frac{1}{\xi^{T}}$ and $\kappa \geq \tau-1$. In this case, the formulas

$\gamma^{\prime}=\frac{1}{\xi^{T}}-\beta$

$\delta=\frac{1}{\xi^{T} \beta}$

coincide with those in M2 (see (53) and (54)). Such a coincidence is not accidental, because the explicit formulas for the critical exponents in models M1 and M2 are very different, and testifies to the existence of connections between the values of critical exponents of different models and properties of a PT curve, whose shape is described by the exponent $\xi^{T}$. This result is of great importance for the further development of model M1 in the context of QCD. By the contemporary state of art, QCD in the case of $2+1$ quark flavors falls in the universality class of the three-dimensional $\mathrm{O}(4)$-symmetric spin model [1214, 40, 41]. At the same time, for $\mathrm{O}(\mathrm{N})$ spin models, $\xi^{T}$ equals the ratio of the magnetic $y_{h}$ and thermal $y_{t}$ exponents [42-44]. For the $\mathrm{O}(4)$-symmetric model, this exponent is connected with the critical exponents $\beta$ and $\delta$ in the same way as in (48), i.e., $\xi^{T}=(\beta \delta)^{-1}[43]$. Therefore, we may assert that M1 in this mode corresponds to QCD in the case of $2+1$ quark flavors. 
T a b l e 2. Parameters of M1, which generate the critical exponents with the values from Table 1 . The values of parameter $\omega$ obtained from the formulas for $\boldsymbol{\alpha}^{\prime}, \boldsymbol{\alpha}_{s}^{\prime}$, and $\boldsymbol{\alpha}_{c}^{\prime}$ are marked by ${ }^{*}$, , and ***, respectively. The symbol $\emptyset$ means that it is impossible to find a value of the relevant parameter, which would allow one to describe the critical exponents from Table 1

\begin{tabular}{|c|c|c|c|c|c|c|}
\hline & $\begin{array}{l}\text { Simple } \\
\text { liquids }\end{array}$ & $\begin{array}{c}\text { 2D model } \\
\text { Ising }\end{array}$ & $\begin{array}{c}\text { 3D model } \\
\text { Ising }\end{array}$ & $\begin{array}{c}\mathrm{O}(2) \text { spin } \\
\text { model }\end{array}$ & $\begin{array}{c}\mathrm{O}(3) \text { spin } \\
\text { model }\end{array}$ & $\begin{array}{c}\mathrm{O}(4) \text { spin } \\
\text { model }\end{array}$ \\
\hline$\chi$ & $\geq 0$ & $\geq 0$ & $\geq 0$ & $\geq 0$ & $\geq 0$ & $\geq 0$ \\
\hline$\tau$ & $1.818(9)$ & $31 / 16$ & $1.8273(5)$ & $1.8270(5)$ & $1.8271(5)$ & $1.828(1)$ \\
\hline$\kappa$ & $\in[0.818(9), 1)$ & $\in[15 / 16,1)$ & $\in[0.8273(5), 1)$ & $\in[0.8270(5), 1)$ & $\in[0.8271(5), 1)$ & $\in[0.828(1), 1)$ \\
\hline$\xi^{T}$ & $0.631(19)$ & $8 / 15$ & $0.6395(9)$ & $0.6002(4)$ & $0.5666(45)$ & $0.55(1)$ \\
\hline$\omega^{*}$ & $\emptyset$ & 0 & $\emptyset$ & $0.0146(8)$ & $0.1336(15)$ & $0.19(6)$ \\
\hline$\omega^{* *}$ & $1.565(18)$ & $\geq 0$ & $1.5639(5)$ & $1.6661(8)$ & $1.7647(15)$ & $1.81(6)$ \\
\hline$\omega^{* * *}$ & $\geq 1.565(18)$ & $\geq 0$ & $\geq 1.5639(5)$ & $\geq 1.6661(8)$ & $\geq 1.7647(15)$ & $\geq 1.81(6)$ \\
\hline
\end{tabular}

T a b l e 3. Parameters of M2, which generate the critical exponents with values from Table 1 . The values of parameter $\omega$, which are obtained by the formulas for $\boldsymbol{\alpha}^{\prime}, \boldsymbol{\alpha}_{s}^{\prime}$, and $\boldsymbol{\alpha}_{c}^{\prime}$, are marked by *, **, and ***, respectively. The symbol $\emptyset$ means that it is impossible to find the value of relevant parameter, which would allow one to describe the critical exponents from Table 1

\begin{tabular}{|c|c|c|c|c|c|c|c|c|c|c|c|c|}
\hline \multirow[b]{2}{*}{$\xi^{T}$} & \multicolumn{2}{|c|}{$\begin{array}{l}\text { Simple } \\
\text { liquids }\end{array}$} & \multicolumn{2}{|c|}{$\begin{array}{c}\text { 2D model } \\
\text { Ising }\end{array}$} & \multicolumn{2}{|c|}{$\begin{array}{c}\text { 3D model } \\
\text { Ising }\end{array}$} & \multicolumn{2}{|c|}{$\begin{array}{c}\mathrm{O}(2) \text { spin } \\
\text { model }\end{array}$} & \multicolumn{2}{|c|}{$\begin{array}{c}\mathrm{O}(3) \text { spin } \\
\text { model }\end{array}$} & \multicolumn{2}{|c|}{$\begin{array}{c}\mathrm{O}(4) \text { spin } \\
\text { model }\end{array}$} \\
\hline & $0.631(19)$ & $0.631(19)$ & $8 / 15$ & $8 / 15$ & $0.6395(9)$ & $0.6395(9)$ & $0.6002(4)$ & $0.6002(4)$ & $0.5666(45)$ & $0.5666(45)$ & $0.55(1)$ & $0.55(1)$ \\
\hline$\zeta^{+}$ & 1 & 1 & 1 & 1 & 1 & 1 & 1 & 1 & 1 & 1 & 1 & 1 \\
\hline$\zeta^{-}$ & 1 & $>1$ & 1 & $>1$ & 1 & $>1$ & 1 & $>1$ & 1 & $>1$ & 1 & $>1$ \\
\hline$\xi^{+}$ & $0.84(1)$ & $0.84(1)$ & 1 & 1 & $0.8902(5)$ & $0.8902(5)$ & $1.0143(4)$ & $1.0143(4)$ & $1.127(30)$ & $1.127(30)$ & $1.21(6)$ & $1.21(6)$ \\
\hline$\xi^{-}$ & $0.84(1)$ & $>0$ & 1 & $>0$ & $0.8902(5)$ & $>0$ & $1.0143(4)$ & $>0$ & $1.127(30)$ & $>0$ & $1.21(6)$ & $>0$ \\
\hline$\omega^{* *}$ & $\geq 0$ & $\geq 0$ & $\geq 0$ & $\geq 0$ & $\geq 0$ & $\geq 0$ & $\geq 0$ & $\geq 0$ & $\geq 0$ & $\geq 0$ & $\geq 0$ & $\geq 0$ \\
\hline$\omega^{* * *}$ & $\geq 0$ & $\geq 0$ & $\geq 0$ & $\geq 0$ & $\geq 0$ & $\geq 0$ & $\geq 0.0146(8)$ & $\geq 0.0146(8)$ & $\geq 0.1336(15)$ & $\geq 0.1336(15)$ & $\geq 0.19(6)$ & $\geq 0.19(6)$ \\
\hline
\end{tabular}

\subsection{Critical exponents of M2}

The same reasoning as that for M1 demonstrates that $\frac{d \mu_{\rho_{\mathrm{cep}}}}{d T} \simeq-\frac{\partial \Delta z}{\partial T} / \frac{\partial \Delta z}{\partial \mu} \sim \frac{d \mu_{\Sigma}}{d T}$ in M2. Just therefore, the shape of the critical isochore at supercritical temperatures is also determined in this model by the parameter $\xi^{T}$. Hence, Eqs. (28) and (29) imply that

$\alpha=\alpha^{\prime}$

in M2. In addition, Eqs. (32) and (33) and the parametrization accepted in M2 allow us to find the other exponents of this model:

$\beta^{+}=1+\xi^{+}-\frac{1}{\xi^{T}}$

$\left.\beta^{-}\right|_{\zeta^{-}=1}=\min \left(1, \frac{1}{\xi^{T}}, 1+\xi^{-}-\frac{1}{\xi^{T}}\right)$

$\left.\beta^{-}\right|_{\zeta^{-}>1}=\min \left(1, \frac{1}{\xi^{T}}\right)$
These formulas and the condition $\beta<\min \left(1, \frac{1}{\xi^{T}}, \chi\right)$ yield $\beta=\beta^{+}$for $\zeta^{-}>1$ and $\beta=\min \left(\beta^{+}, 1+\xi^{-}-\frac{1}{\xi^{T}}\right)$ for $\zeta^{-}=1$. In addition, model M2 yields

$\gamma^{\prime}=\frac{1}{\xi^{T}}-\beta$

$\delta=\frac{1}{\xi^{T} \beta^{+}}$

The explicit formulas for the critical exponents in M2 are very different from those in M1. Moreover, as distinct from M1, the exponents $\tau, \kappa$, and $\chi$ do not generally enter formulas (50)-(54). Such a property of M2 is extremely interesting and exotic, because the critical exponents in all other models involving the Fisher exponent $\tau$ (i.e., in M1, MSMF [24, 34], and FDM [25]) depend on $\tau$. However, as it is seen from Table 3, this model is quite able to describe the critical exponents of the two- and three-dimensional Ising models, simple 
liquids, and three-dimensional $\mathrm{O}(2)-, \mathrm{O}(3)$-, and $\mathrm{O}(4)-$ symmetric spin models.

It was mentioned in Section 3.1 that, despite the significant distinctions of the explicit formulas for critical exponents in M1 and M2, there exist the modes, where the relations between exponents are completely identical in both models. In M2, such a mode is realized for $\zeta^{-}>1$ or for $\zeta^{-}=1$ and $\xi^{-}>\xi^{+}$, where formulas (53) and (54) in M2 completely coincide with the analogous formulas (47) and (48) in M1. In this case, like that in $\mathrm{M} 1, \xi^{T}=(\beta \delta)^{-1}$, which takes place for the $\mathrm{O}(4)$ symmetric model [43] and, hence, for QCD in the case of $2+1$ quark flavors [12-14, 40,41]. Such a connection between models M1 and M2, on the one hand, and the parameters of lattice QCD, on the other hand, seems to be not accidental and it ensures the possibility to reliably simulate not only the equation of state of a strongly interacting substance with the help of M1 and M2, but also such nontrivial properties as the QCD matter critical exponents at TCP $/ \mathrm{CP}$.

\section{Scaling Relations}

The well-known scaling laws

$\alpha^{\prime}+2 \beta+\gamma^{\prime} \geq 2$

$\alpha^{\prime}+\beta(1+\delta) \geq 2$

$\gamma^{\prime}+\beta(1-\delta) \geq 0$

play traditionally the significant role in the systematization of universality classes [2,3] and in the theory of critical phenomena [5,42]. For simple liquids, these laws were proved by Fisher [2], Griffiths [45], and Liberman [46], respectively. For magnetic systems, these laws are called the Rushbrooke, Griffiths and Widom relations respectively.

However, the situation concerning the validity of the scaling laws for real physical systems is not so trivial, as it seems at first glance. For example, there exist the models [3,34], where relations (55) and (56) are not satisfied in the general case. Moreover, some experimental works (e.g., [6] or [7,8]) indicated the difficulties concerning the validity of the Fisher and Griffiths laws. For example, some unsolved problems exist even in the case of the well-defined critical exponent $\beta$ [7]. Its experimentally determined value $\beta=0.300$ (4) for a binary ionic solution $\mathrm{PeNBr}-\mathrm{H}_{2} \mathrm{O}$ does not agree with the other data on simple liquids and with the Ising model. In addition, it leads to liquids and with the Ising model. In addition, it leads to the critical exponents for simple fluids: $\alpha^{\prime}+2 \beta+\gamma^{\prime}=1.95<2$. In this case, the situation concerning the Griffiths relation for simple fluids looks more dramatic, since $\alpha^{\prime}+\beta(1+\delta)=1.75<2$, if the value of $\beta$ is taken from work [7]. It is worth noting that, on the basis of available data, it is possible to assert that these laws hold in the limits of experimental errors, but these errors are very large sometimes. Just therefore, it is quite interesting to verify the validity of the scaling laws (55)-(57) for critical exponents in M1 and M2.

It is convenient to start with the analysis of the Liberman relation. In both versions of QGBM, the $\mathrm{O}(4)$ scaling mode is realized with the relations $\gamma^{\prime}=\frac{1}{\xi^{T}}-\beta$ and $\delta=\frac{1}{\xi^{T} \beta}$. It is easy to verify that, in this mode, relation (57) holds as the equality. Moreover, model M1 includes the mode for $\chi=0$, which reproduces the critical exponents $\beta, \gamma^{\prime}$, and $\delta$ in the same form as that in MSMF [34]. Therefore, like the case of MSMF, the Liberman scaling relation is valid in M1 always in this case. We now demonstrate this, by using the explicit formulas for the relevant exponents:

$\left[\gamma^{\prime}+\beta(1-\delta)\right]_{\mathrm{M} 1}=\frac{\min \left(0, \frac{\zeta}{\kappa} \min (\tau-1, \kappa)-\frac{1}{\xi^{T}}\right)}{\tau-2} \geq 0$

Here, we used $\min (0, \ldots) \leq 0$ and $\tau<2$. The validity of relation (57) in M1 in all other cases can be proved in the same way. For M2, the situation is completely analogous. The explicit formulas for the critical exponents in this model and the relation $\beta \leq \beta^{+}$yield

$\left[\gamma^{\prime}+\beta(1-\delta)\right]_{\mathrm{M} 2}=\frac{1}{\xi^{T}}\left(1-\frac{\beta}{\beta^{+}}\right) \geq 0$.

Using the proved Liberman inequality, we can show now that the relation $\alpha^{\prime}+\beta(\delta+1) \leq \alpha^{\prime}+2 \beta+\gamma^{\prime}$ holds always for M1 and M2. It is the important result with several consequences. First, we may assert on this basis that the conditions for the Fisher law (55) and the Griffiths one (56) to be valid coincide only under the condition that the Liberman law is satisfied as the equality. Second, this relation demonstrates that even if relation (55) holds, the validity of relation (56) is not guaranteed in the general case. Using the explicit formulas for the critical exponents $\alpha^{\prime}, \beta$, and $\gamma^{\prime}$, we can show that, in M1,

$\left[\alpha^{\prime}+2 \beta+\gamma^{\prime}\right]_{\mathrm{M} 1}=2+\frac{\zeta}{\kappa}-2 \min \left(1, \frac{1}{\xi^{T}}\right)<2$ 
for $\frac{\zeta}{\kappa}<2 \min \left(1, \frac{1}{\xi^{T}}\right)$. In M2, we have that

$\left[\alpha^{\prime}+2 \beta+\gamma^{\prime}\right]_{\mathrm{M} 2}=2 \max \left(1, \frac{1}{\xi^{T}}\right)-\gamma_{\mathrm{M} 2}^{\prime}$

is obligatorily less than 2 for $\frac{1}{\xi^{T}}<1+\frac{\gamma_{\mathrm{M} 2}^{\prime}}{2}$. Thus, we may assert that the Fisher relation is not valid in models M1 and M2 in the general case for the traditional definition of $\alpha^{\prime}$. It is worth noting that, according to the abovepresented conclusions, the Griffiths relation (56) is not satisfied also in both versions of QGBM with surface tension.

The appearance of difficulties concerning the validity of the scaling laws including the exponent $\alpha^{\prime}$ (i.e., the Fisher and Griffiths relations) was established else in [3]. To explain this fact, it was assumed that the definition of this exponent was not proper. In order that the scaling inequalities (55) and (56) be satisfied, the new exponent $\alpha_{s}^{\prime}$ describing the behavior of the difference of the heat capacities of two pure phases on the PT curve was introduced:

$\Delta C \equiv\left(C^{+}-C^{-}\right)_{T=T_{c}} \sim|t|^{-\alpha_{s}^{\prime}}$.

Using the definition of the heat capacities of pure phases, $C=\frac{T}{\rho} \frac{d s}{d T}$, and the Clapeyron-Clausius equation, $\Delta C$ can be presented in the form

$$
\frac{\Delta C}{T_{c}}=\left[\frac{\rho^{-}-\rho^{+}}{\rho^{+} \rho^{-}} \frac{d\left(s_{Q}+\frac{d \mu_{c}}{d T} \rho_{Q}\right)}{d T}-\frac{d \mu_{c}}{d T} \frac{d \ln \frac{\rho^{+}}{\rho^{-}}}{d T}\right]_{T=T_{c}},
$$

which is valid both for M1 and M2. Since $\rho^{+}-\rho^{-} \sim|t|^{\beta}$ on the PT curve, relations (11) and (26) imply that the first term in the formula for $\Delta C$ behaves in a neighborhood of the critical point as $|t|^{\min \left(\omega, \frac{1}{\xi^{T}}-2\right)+\beta}$. Using the approximation $\ln \frac{\rho^{+}}{\rho^{-}} \simeq \frac{\rho^{+}-\rho^{-}}{\rho^{-}}$, which is proper for the small deviations of the density from the critical value, it is possible to show that the behavior of the second term is described by the expression $|t| \sim \frac{1}{\xi^{T}}-2+\beta$. Hence,

$\alpha_{s}^{\prime}=\max \left(\frac{1}{\xi^{T}}-\omega, 2\right)-\beta-\frac{1}{\xi^{T}}$

in both versions of QGBM with surface tension. Formula (64) allows one to directly verify the Fisher hypothesis and to answer the question: Can the scaling laws be satisfied with the use of the exponent $\alpha_{s}^{\prime}$ instead of $\alpha^{\prime}$ ? In both models, this hypothesis does work for the Fisher law. In order to prove it for model M1, we use the relation $\beta_{\mathrm{M} 1} \leq \frac{\zeta}{\kappa}(2-\tau+\min (\kappa, \tau-1))-\frac{1}{\xi^{T}}$, which follows from the explicit expression (43) for this exponent, and obtain $\frac{\zeta}{\kappa}-\beta-\frac{1}{\xi^{T}} \geq 0$. Hence,

$\left[\alpha_{s}^{\prime}+2 \beta+\gamma^{\prime}\right]_{\mathrm{M} 1}=\max \left(2, \frac{1}{\xi^{T}}\right)+\frac{\zeta}{\kappa}-\beta-\frac{1}{\xi^{T}} \geq 2$,

where the obvious inequality $\max (2, \ldots) \geq 2$ was used on the second step. With the help of (53) and (64), it is possible to show that the critical exponents in M2 satisfy the relation

$\left[\alpha_{s}^{\prime}+2 \beta+\gamma^{\prime}\right]_{\mathrm{M} 2}=\max \left(2, \frac{1}{\xi^{T}}\right) \geq 2$.

As it is seen, the redefinition of the critical exponent $\alpha^{\prime}$ according to the Fisher definition (62) does allow us to satisfy the scaling law (55) in both versions of QGBM with surface tension. However, this is not valid for the scaling law (56). Indeed, model M2 meets certain problems concerning the validity of the Griffiths relation

$\left[\alpha_{s}^{\prime}+\beta(1+\delta)\right]_{\mathrm{M} 2}=\max \left(\frac{1}{\xi^{T}}-\omega, 2\right)+\frac{1}{\xi^{T}}\left(\frac{\beta}{\beta^{+}}-1\right)$,

which is less than 2 for $\beta=\beta^{-}<\beta^{+} \min \left(1,(2+\omega) \xi^{T}\right)$. Thus, the hypothesis proposed by Fisher is not valid for the Griffiths relation (56). We note that, from the viewpoint of the validity of scaling relations, the reason for the failure of the Fisher definition of $\alpha_{s}^{\prime}$ consists in the following. In the course of calculations of $\Delta C$, the most singular terms can be cancelled if they are referred to two phases of the same type. Just this happens in M2, since hadrons and QGP, which coexist on both sides of the deconfinement PT curve are the gas phases. We note that the validity of the scaling laws with $\alpha_{s}^{\prime}$ in M1 is related to the circumstance that, in this model, the deconfinement transition is PT from the gaseous hadronic phase to the QGL phase. We now prove that any definition of the critical exponent $\alpha^{\prime}$ will not allow one to solve the problem with the traditional formulation of the scaling Griffiths relation (56). From the viewpoint of the validity of the scaling laws, the critical exponent $\alpha_{c}^{\prime}$, being a generalization of the Fisher definition (62), seems more suitable than $\alpha_{s}^{\prime}$. We have

$C_{\text {tot }} \sim|t|^{-\alpha_{c}^{\prime}}$

where $C_{\text {tot }}=\left[K^{+} C^{+}+K^{-} C^{-}\right]_{T=T_{c}}$. The coefficients $K^{+}$and $K^{-}$, which are bounded for all values of the 
temperature and the baryon chemical potential, are related to the contributions of pure phases to the total heat capacity of the system in a neighborhood of the critical point. It is clear that, by choosing a specific functional dependence of these coefficients on $T$ and $\mu$, various values of exponent $\alpha_{c}^{\prime}$ can be realized. For example, $K^{+}=1$ and $K^{-}=-1$ for the Fisher definition of $\alpha_{s}^{\prime}$. The more suitable and physically substantiated definition corresponds to positive $K^{+}$and $K^{-}$, which allows one to prevent the cancellation of any terms in formulas for the heat capacities of two phases, $C^{+}$and $C^{-}$. In addition, this corresponds to the physical content of the heat capacity $C_{\text {tot }}$ characterizing the system, which exists in the state of the low-temperature or hightemperature phase due to the significant fluctuations in a neighborhood of TCP or CP with certain probabilities. Just therefore, while determining $\alpha_{c}^{\prime}$, the contributions from each phase can be analyzed separately. With this purpose, we represent the heat capacities of pure phases on the $\mathrm{PT}$ curve in the form

$C^{ \pm}=\frac{T}{\rho^{ \pm}}\left[\frac{d s_{Q}}{d T}-\frac{d}{d T}\left(\left(\rho^{ \pm}-\rho_{Q}\right) \frac{d \mu_{c}}{d T}\right)\right]$.

We note that this formula is a consequence of the Clapeyron-Clausius equation $\frac{d \mu_{c}}{d T}=-\left.\frac{s^{ \pm}-s_{Q}}{\rho^{ \pm}-\rho_{Q}}\right|_{T=T_{c}}$ for the gaseous phases (hadrons and QGP) and becomes the identity for the QGL phase, since $\rho=\rho_{Q}$ in the last case. Using (69) and the proposed definition (68), we can find $C_{\text {tot }}$. However, this should not be done, since we can separately analyze the behavior of the heat capacities of pure phases by virtue of the positiveness of the coefficients $K^{+}$and $K^{-}$. In model M1, QGL is the high-temperature phase, and, hence, the formula for its heat capacity includes only the first term from (69). Relation (26) implies that the behavior of QGL in a neighborhood of TCP, like the behavior of the first term in the formula for the heat capacity of the low-temperature phase, is described by the power $|t|^{\omega}$. Taking the relation $\left(\rho^{-}-\rho_{Q}\right)_{T=T_{c}} \sim|t|^{\beta}$ into account and using (11), we can show that the second term in the formula for $C^{-}$behaves as $|t|^{\frac{1}{\xi^{T}+\beta-1}}$. The critical exponent $\alpha_{c}^{\prime}$ describes the behavior of the principal term in the formula for $C_{\mathrm{tot}}$. Therefore,

$\alpha_{c}^{\prime}=\max \left(\frac{1}{\xi^{T}}+\beta-\omega, 2\right)-\beta-\frac{1}{\xi^{T}}$.

To determine the exponent $\alpha_{c}^{\prime}$ in M2, we note that the linear combination of the first terms in the formulas for $C^{ \pm}$behaves as $|t|^{\omega}$ in a neighborhood of CP. In addition, using the definition of the baryon densities $\rho^{ \pm}$and $\rho_{Q}$ as the derivatives of the relevant pressures with respect to $\mu$ and Eqs. (8) and (9), we obtain the following relation for the gas phases, which coexist on both sides of the PT curve:

$\left(\rho^{ \pm}-\rho_{Q}\right)_{T=T_{c}}=\left.T_{c} \frac{A_{\mu}-u \frac{\partial \sigma^{ \pm}}{\partial \mu} I_{\tau-\kappa}}{1-\frac{\partial F_{H}}{\partial z}+u I_{\tau-1}}\right|_{T=T_{c}}$

Parametrizations (10), (15), and (18) imply that, in a neighborhood of CP, the behavior of $\left(\rho^{ \pm}-\rho_{Q}\right)_{T=T_{c}}$ is described by the expression $|t|^{\min \left(\chi, \beta^{ \pm}\right)}$for $\zeta^{ \pm}=1$ or by $|t|^{\chi}$ for $\zeta^{ \pm}>1$. We recall that $\zeta^{+}=1$ in M2 in all cases. Using the explicit expression for the exponent $\beta$ in the present model, we can show that the linear combination of other terms from the formulas for $C^{+}$and $C^{-}$behaves as $|t|^{\beta+\frac{1}{\xi^{T}}-2}$. The above analysis allows us to determine the exponent $\alpha_{c}^{\prime}$ in model M2. The expression for it completely coincides with (70).

The explicit expressions (64) and (70) demonstrate that $\alpha_{c}^{\prime} \geq \alpha_{s}^{\prime}$. Therefore, the definition of the exponent $\alpha_{c}^{\prime}$ does look more adequate relative to the validity of the scaling laws. However, it does not allow us to satisfy the Griffiths scaling relation in the form (56). For example, in model M2, the combination of the critical exponents corresponding to this relation can be less than 2 for $\beta=$ $\beta^{-}<\beta^{+}$and $\beta \leq 2+\omega-\frac{1}{\xi^{T}}$. Indeed, $\alpha_{c}^{\prime}=2-\beta-\frac{1}{\xi^{T}}$ in this mode, and, hence,

$\left[\alpha_{c}^{\prime}+\beta(1+\delta)\right]_{\mathrm{M} 2}=2+\frac{1}{\xi^{T}}\left(\frac{\beta}{\beta^{+}}-1\right)<2$.

Thus, even the most physically substantiated definition of the critical exponent $\alpha_{c}^{\prime}$ does not allow us to satisfy the scaling laws in the general case. In order to demonstrate the absence of a definition allowing one to make it, we consider also the exponent $\alpha_{m}^{\prime}$ defined by

$\frac{T_{c}}{\rho_{\text {tot }}} \frac{d s_{\text {tot }}}{d T} \sim|t|^{-\alpha_{m}^{\prime}}$

where the entropy $s_{\text {tot }}=\left[K^{+} s^{+}+K^{-} s^{-}\right]_{T=T_{c}}$, and $\rho_{\text {tot }}$ is the baryon density corresponding to it. We note that the coefficients $K^{+}$and $K^{-}$should satisfy the same conditions as those in the case of the definition of $C_{\text {tot }}$ by the reasons analogous to the above-indicated ones. The derivative of $s_{\text {tot }}$ contains the terms proportional to $\frac{d s^{+}}{d T}$ and $\frac{d s^{-}}{d T}$, and their behavior is described by the expression $|t|^{-\alpha_{c}^{\prime}}$. Parametrizing the temperature dependence of $K^{+}$and $K^{-}$in a neighborhood of the critical point by $K^{ \pm} \sim|t|^{\eta^{ \pm}}$, we obtain that the terms in the expression for $\frac{d s_{\text {tot }}}{d T}$, which are proportional to the derivatives of these coefficients, behave as $|t|^{\min \left(\eta^{+}, \eta^{-}\right)-1}$. We note 
that, as $\eta^{+} \rightarrow 0$ and $\eta^{-} \rightarrow 0$, their behavior is the most singular. Therefore, for the largest value of exponent $\alpha_{m}^{\prime}$, we obtain $\alpha_{m}^{\prime}=\max \left(1, \alpha_{c}^{\prime}\right)$. In addition, the explicit formulas for the exponents $\alpha_{c}^{\prime}$ and $\beta$ yield $\alpha_{c}^{\prime}<1$. Hence, we have finally:

$\alpha_{m}^{\prime}=1$.

The same result for the largest value of $\alpha^{\prime}$ was obtained in work [11]. Just this circumstance allows us to assert that the exponent $\alpha_{m}^{\prime}$ defined according to (73) is the most suitable in order to satisfy the scaling relation. One more argument to the favor of this assertion is the relation between the critical exponents $\alpha^{\prime}, \alpha_{s}^{\prime}, \alpha_{c}^{\prime}$, and $\alpha_{m}^{\prime}$ :

$\alpha^{\prime} \leq \alpha_{s}^{\prime} \leq \alpha_{c}^{\prime}<\alpha_{m}^{\prime}$

which follows directly from formulas (27), (64), (70), and (74). It is quite possible that such a hierarchy holds not only for QGBM with surface tension, but also for other models and physical systems. However, this cannot be verified at the present time, unfortunately, due to the absence of the comprehension of experimental data. However, if it is true, then the violation of the scaling laws with the critical exponent $\alpha_{m}^{\prime}$ is the unambiguous proof of the existence of new non-Fisher universality classes, where such laws take the forms different from (55)-(57). We now verify whether this happens in the case of the Griffiths scaling law

$\alpha_{m}^{\prime}+\beta(1+\delta)=1+\beta(1+\delta)$,

which is not satisfied in the form $(56)$ for $\beta(1+\delta)<1$. In model M2, this holds for $\beta=\beta^{-}<\beta^{+}$and $\beta^{-}<$ $\frac{\xi^{T} \beta^{+}}{1+\xi^{T} \beta^{+}}$. The presented example demonstrates that any, even commonly accepted, definition of the critical exponent $\alpha^{\prime}$ does not allow one to satisfy the Griffiths scaling relation in the traditional form. Since this violation of the scaling happens in the model, which contains no defects and is completely consistent with requirements of the Fisher theorem [2], it is necessary to clarify whether the requirements of this theorem can be supplemented so that it would be always valid for a certain class of physical systems. In works $[10,11]$ on the basis of the analysis of the quite general requirements of stability of the critical state of the system, it was assumed that the Fisher and Griffiths scaling laws hold only under the condition of the equality of the critical exponents $\alpha$ and $\alpha^{\prime}$. Therefore, it is natural to verify whether this condition together with the requirements of the Fisher theorem lead to the validity of the scaling relations in the forms (55) and (56) in QGBM with surface tension. The explicit formulas for $\alpha,(40)$ and (41) in M1 and (49) in M2, demonstrate that both versions of QGBM realize the mode, where $\alpha=\alpha^{\prime}$. In M1, this happens for $\chi \neq 1-\frac{1}{\xi^{T}}$, which does not ensure the validity of the Fisher (55) and Griffiths (56) scaling laws. In M2, the equality $\alpha=\alpha^{\prime}$ holds generally without the imposition of additional conditions on any model parameters. Hence, the equality of the critical exponents $\alpha$ and $\alpha^{\prime}$ does not ensure the validity of the scaling laws (55) and (56) in the general case.

Since neither the redefinition of the critical exponent $\alpha^{\prime}$ [3], nor the imposition of the additional condition $\alpha=\alpha^{\prime}$ [11] allow one to satisfy the scaling law (56) in QGBM with surface tension in the general case, we make conclusion that QGBM belongs to a new (nonFisher) universality class, in which the Griffiths relation has the form different from (56). Therefore, it is possible to conclude that the scaling problem is more profound as compared with the thoughts of the previous researchers about it and can require the reformulation of the scaling laws for some physical systems. As was mentioned above, their violation occurs namely in the models, where the types of the phases coexisting on both sides of the PT curve are identical. The example of such a model is given by M2. Since the violation of the scaling in M2 for all definitions of $\alpha^{\prime}$ is related to the Griffiths relation and occurs for $\beta=\beta^{-}<\beta^{+}$, this relation should be modified so that it would be true for arbitrary values of $\beta$. The explicit formulas for the appropriate critical exponents demonstrate that the relation

$\alpha_{c}^{\prime}+\beta+\beta^{+} \delta \geq 2$,

which is an analog of (56), is always satisfied in M1 and M2. This relation is written with the use of the critical exponent $\alpha_{c}^{\prime}$, because it is the most suitable from the viewpoint of the validity of the scaling and simultaneously, as distinct from $\alpha_{m}^{\prime}$, is physically substantiated. However, at least in M1 and M2, relation (77) is also satisfied with the use of $\alpha_{s}^{\prime}$, whereas this inequality does not hold for the traditional definition of $\alpha^{\prime}$. In addition, the condition $\beta \leq \beta^{+}$implies that, in the case where inequality (56) is satisfied for a certain physical system, relation (77) is satisfied automatically, but the opposite is not true. The unique property of M2, which allowed us to arrive at the necessity to modify the Griffiths scaling law, consists in the following. In this model, the mode, where $\beta=\beta^{-}<\beta^{+}$, is generated only at the imposition of such conditions on the model parameters that are inconsistent with this relation. For example, the relation $\beta^{-}<\beta^{+}$in M1 is an automatic consequence of 
the general requirement of boundedness of the volume fraction of the hadronic phase at TCP. In MSMF and in the spin and magnetic systems, this relation follows generally from the fact that the value of a quantity in the high-temperature phase, whose jump is described by the critical exponent $\beta$ (the density of the nuclear matter or the spontaneous magnetization) coincides with the critical one. Indeed, for these systems, $\rho^{+}=\rho_{\text {cep }}=\frac{1}{b}$ [24], where $b$ stands for the volume of one nucleon, and $M^{+}=M_{\text {cep }}=0$. Therefore, according to definition (30), the exponent $\beta^{+}$is formally equal in these cases to infinity, since $0<|t|<1$ on the PT curve.

\section{Conclusions}

The extremely wide application of the hypothesis of similarity to the critical behavior of various physical systems leads to the necessity of the detailed consideration of the question about the association of these systems with various universality classes. The traditional scaling laws (55)-(57) [2,3] hold for a quite wide spectrum of physical systems and models, which belong to the Fisher universality classes. However, there exist the examples where this pattern does not true.

With the purpose to answer the question about the nature of such a violation, we have performed the comprehensive analysis of QGBM with surface tension. We have considered its versions, which generate the phase diagrams with TCP and CP. To attain a high generality of the model, we have introduced three new parameters. They are $\xi^{T}$, which determines the shape of the PT curve in the $\mu-T$ coordinates, $\chi$ introduced due to the validity of the Clapeyron-Clausius equation on this curve, and $\omega$, with the help of which the behavior of the entropy density of the liquid phase is described. The introduction of these exponents has allowed us to completely describe the critical exponents of various physical systems and models.

The critical exponents $\alpha, \alpha^{\prime}, \beta, \gamma^{\prime}$, and $\delta$ of QGBM with surface tension have been found as functions of the model parameters. Despite the significant similarity of M1 and M2, their critical exponents, except for $\alpha^{\prime}$, are very different. This can be explained by the difference of the mechanisms of generation of critical points in these models. However, we have established the existence of modes, where the relations between all critical exponents of these models are identical. In addition, we have demonstrated that the solutions of MSMF [34] enter into M1, as a partial case. This fact should be considered as the undoubtful proof of the generality of QGBM with surface tension. The obtained formulas for critical ex- ponents allow us to directly verify the validity of scaling laws in the form (55)-(57). We have established that, in both versions of QGBM with surface tension, the Fisher (55) and Griffiths (56) scaling relations including the exponent $\alpha^{\prime}$ are violated in the general case, whereas the Liberman law (57) holds in all cases. For the further study of this problem, we have verified the Fisher hypothesis [3] and have determined the critical exponent $\alpha_{s}^{\prime}$. The analysis of the scaling laws with this exponent for models M1 and M2 allows us to clarify the conditions, under which it is fruitful. We have revealed that, in the general case, this hypothesis is suitable only for models, where the types of phases existing on different sides of the PT curve are different. By analyzing the reasons for the failure of the Fisher definition of $\alpha_{s}^{\prime}$, we have introduced the new critical exponent $\alpha_{c}^{\prime}$, which is more suitable from the viewpoint of the validity of the Fisher (55) and Griffiths (56) scaling relations. In addition, we have introduced the definition of the exponent $\alpha_{m}^{\prime}$ with the purpose to attain the completeness of the analysis. It is worth noting that this exponent possessing the largest value among all $\alpha^{\prime}$ is not physically substantiated. In other words, its introduction is caused by purely methodical reasons. Therefore, with regard for the clear physical content of the heat capacity $C_{\text {tot }}$, we insist that namely the critical exponent $\alpha_{c}^{\prime}$ introduced by us is the most suitable and physically substantiated. As the unexpected result, we indicate the circumstance that none of the definitions of $\alpha_{s}^{\prime}, \alpha_{c}^{\prime}$, and $\alpha_{m}^{\prime}$ allows one to satisfy at once all scaling relations $(55)-(57)$ in the general case. This circumstance testifies clearly to the existence of models, M2 in the case under consideration, for which these relations take the forms different from (55)-(57). We note that this conclusion is supported by the fact that, contrary to $[10,11]$, the imposition of the additional condition $\alpha=\alpha^{\prime}$ does not lead to the solution of the scaling problem both in M1 and M2. We hope for that the future experimental studies performed in a consistent way will allow one to finally clarify this question. It is worth noting that the measurements should be carried out for a single substance to ensure the precision of the results.

Though the results obtained require the further studies, they allow us to assert the existence of non-Fisher universality classes, for which some of the scaling laws can be different from their traditional forms (55)-(57). On the basis of the analysis of models M1 and M2, we have proved the existence of one of such relations (77).

The authors are sincerely grateful to I.M. Mishustin and L.M. Satarov for the fruitful discussion of results and 
T.V. Ivanchuk for her essential help in the preparation of the manuscript to the publication.

1. E.A. Guggenheim, J. Chem. Phys. 13, 253 (1945).

2. M.E. Fisher, J. Math. Phys. 5, 944 (1964).

3. M.E. Fisher and B.U. Felderhof, Ann. Phys. 58, 217 (1970).

4. K. Huang, Statistical Mechanics (Wiley, New York, 1987).

5. H.E. Stanley, Introduction to Phase Transitions and Critical Phenomena (Clarendon Press, Oxford, 1971).

6. P.R. Roach, Phys. Rev. 170, 213 (1968); P.R. Roach and D.H. Douglass, Phys. Rev. Lett. 19, 287 (1967).

7. Yu.I. Shimansky, E.T. Shimanskaya, Int. J. Thermophys. 17, 651 (1996)

8. Yu.I. Shimansky, O.T. Shimans'ka, A.V. Oliinykova, Nauk. Zap. NAU KMA Fiz., 5, 6 (1998).

9. M. Campostrini, A. Pelissetto, P. Rossi, and E. Vicari, Phys. Rev. E 65, 066127 (2002).

10. E.D. Soldatova, Cond. Mat. Phys. 2, 603 (1999).

11. E.D. Soldatova and O.M. Galdina, Cond. Mat. Phys. 8, 793 (2005)

12. F. Karsch, AIP Conf. Proc. 602, 323 (2001).

13. R. Pisarski and F. Wilczek, Phys. Rev. D 29, 338 (1984).

14. F. Wilczek, Int. J. Mod. Phys. A 7, 3911 (1992); F. Wilczek and K. Rajagopal, Nucl. Phys. B 399, 395 (1993)

15. E.V. Shuryak, Prog. Part. Nucl. Phys. 62, 48 (2009).

16. M. Stephanov, Pos LAT2006:024 (2006).

17. P.N. Meisinger and M.C. Ogilvie, Phys. Lett. B 379, 163 (1996); P.N. Meisinger, T.R. Miller, and M.C. Ogilvie, Phys. Rev. D 65, 034009 (2002); A. Mocsy, F. Sannino, and K. Tuominen, Phys. Rev. Lett. 92, 182302 (2004);

18. E. Megias, E. Ruis Arriola, and L.L. Salcedo, Phys. Rev. D 74, 065005 (2006); C. Ratti, M.A. Thaler, and W. Weise, Phys. Rev. D 73, 014019 (2006); C. Sasaki, B. Friman, and K. Redlich, Phys. Rev. D 75, 074013 (2007)

19. K. Fukushima, Phys. Lett. B 591, 277 (2004).

20. B.-J. Schaefer, J.M. Pawlowzki, and J. Wambach, Phys. Rev. D 76, 074023 (2007); T.K. Herbst, J.M. Pawlowski, and B.-J. Schaefer, Phys. Lett. B 696, 58 (2011).

21. M.I. Gorenstein, V.K. Petrov, and G.M. Zinovjev, Phys. Lett. B 106, 327 (1981).

22. I. Zakout and C. Greiner, arXive:1002.3119 [nucl-th].

23. K.A. Bugaev, Phys. Rev. C 76, 014903 (2007).

24. J.P. Bondorf, A.Z. Botvina, A.Z. Iljinov, I.N. Mishustin, and K. Sneppen, Phys. Rep. 257, 133 (1995).

25. M.E. Fisher, Physics 3, 255 (1967).
26. K.A. Bugaev, Phys. Atom. Nucl. 71, 1615 (2008).

27. K.A. Bugaev, V.K. Petrov, and G.M. Zinovjev, Phys. Part. Nucl. Lett. 9, 397 (2012).

28. R. Hagedorn, Nuovo Cim. Suppl. 3, 147 (1965).

29. K.A. Bugaev, L. Phair, and J.B. Elliott, Phys. Rev. E 72, 047106 (2005); K.A. Bugaev and J.B. Elliott, Ukr. J. Phys. 52, 301 (2007).

30. J. Hosek, Czech. J. Phys. 41, 1037 (1991).

31. J. Hosek, Czech. J. Phys. 43, 309 (1993).

32. K.A. Bugaev and G.M. Zinovjev, Nucl. Phys. A 848, 443 (2010).

33. K.A. Bugaev, Phys. Part. Nucl. Lett. 8, 907 (2011).

34. P.T. Reuter and K.A. Bugaev, Phys. Lett. B 517, 233 (2001).

35. A.I. Ivanytskyi, Nucl. Phys. A 880, 12 (2012).

36. M. Campostrini, M. Hasenbusch, A. Pelissetto, P. Rossi, and E. Vicari, Phys. Rev. B 63, 214503 (2001).

37. M. Campostrini, M. Hasenbusch, A. Pelissetto, P. Rossi, and E. Vicari, Phys. Rev. B 65, 144520 (2002).

38. G. Baker, D. Meiron, and B. Nickel, Phys. Rev. B 17, 1365 (1978).

39. K. Kanaya and S. Kaya, Phys. Rev. D 51, 2404 (1995).

40. S. Ejiri et al., Phys. Rev. D 80, 09450 (2009).

41. O. Kaczmarek et al., Phys. Rev. D 83, 014504 (2011).

42. D.A. Lavis and G.M. Bell, Statistical Mechanics of Lattice Systems, Vol. 1 (Springer, Berlin, 1999).

43. F. Karsch, Prog. Theor. Phys. Suppl. 186, 479 (2010).

44. J. Engels and F. Karsch, 1105.0584 [hep-lat].

45. R.B. Griffiths, J. Chem. Phys. 43, 1958 (1965).

46. D.A. Liberman, J. Chem. Phys. 44, 419 (1966).

Received 16.07.12. Translated from Ukrainian by V.V. Kukhtin

ПРО МОЖЛИВІСТЬ ІСНУВАННЯ НЕФІШЕРІВСЬКИХ КЛАСІВ УНІВЕРСАЛЬНОСТІ

О.І. Іваницъкий, К.О. Бугаєв

$\mathrm{P}$ е $з$ ю м е

Критичні показники $\alpha, \alpha^{\prime}, \beta, \gamma^{\prime}$ та $\delta$ моделі кварк-глюонних мішків з поверхневим натягом знайдено як функції найбільш загальних модельних параметрів. Розглянуто два різновиди моделі, які генерують фазову діаграму сильновзаємодіючої матерії з критичною або трикритичною точкою відповідно. Аналіз співвідношень між критичними індексами (скейлінгових законів) демонструє, що у загальному випадку скейлінг може бути порушено. Проаналізовано питання про можливе відновлення 
скейлінгових законів за допомогою фішерівського означення $\alpha_{s}^{\prime}$ та його узагальнень $\alpha_{c}^{\prime}, \alpha_{m}^{\prime}$. Показано, що скейлінгове співвідношення Фішера можна відновити за допомогою узагальнень $\alpha_{c}^{\prime}$ та $\alpha_{m}^{\prime}$, в той час, як жодне означення $\alpha^{\prime}$ нездатне відновити співвідношення Гріффітса у традиційній формі. Також явно показано, що додаткової умови $\alpha=\alpha^{\prime}$ недостатньо для відновлення співвідношення Гріффітса у традиційному вигля- ді. Запропоновано узагальнення цього скейлінгового співвідношення, яке справедливе для всіх відомих моделей. Отримані результати дозволяють нам зробити висновок про можливе існування нефішерівських класів універсальності, для яких традиційні скейлінгові співвідношення можуть бути порушені, у той час, як для них виконуються узагальнені скейлінгові закони. 